\title{
Hong-Ou-Mandel heat noise in the quantum Hall regime
}

Ronetti, Flavio; Vannucci, Luca; Ferraro, Dario; Jonckheere, Thibaut; Rech, Jérôme; Martin, Thierry; Sassetti, Maura

Published in:

Physical Review B

Link to article, DOI:

10.1103/PhysRevB.99.205406

Publication date:

2019

Document Version

Publisher's PDF, also known as Version of record

Link back to DTU Orbit

Citation (APA):

Ronetti, F., Vannucci, L., Ferraro, D., Jonckheere, T., Rech, J., Martin, T., \& Sassetti, M. (2019). Hong-OuMandel heat noise in the quantum Hall regime. Physical Review B, 99(20), [205406].

https://doi.org/10.1103/PhysRevB.99.205406

\section{General rights}

Copyright and moral rights for the publications made accessible in the public portal are retained by the authors and/or other copyright owners and it is a condition of accessing publications that users recognise and abide by the legal requirements associated with these rights.

- Users may download and print one copy of any publication from the public portal for the purpose of private study or research.

- You may not further distribute the material or use it for any profit-making activity or commercial gain

- You may freely distribute the URL identifying the publication in the public portal

If you believe that this document breaches copyright please contact us providing details, and we will remove access to the work immediately and investigate your claim 


\title{
Hong-Ou-Mandel heat noise in the quantum Hall regime
}

\author{
Flavio Ronetti, ${ }^{1,2,3,{ }^{*}}$ Luca Vannucci, ${ }^{1,2,4}$ Dario Ferraro, ${ }^{1,2,5}$ Thibaut Jonckheere, ${ }^{3}$ Jérôme Rech, ${ }^{3}$ \\ Thierry Martin, ${ }^{3}$ and Maura Sassetti ${ }^{1,2}$ \\ ${ }^{1}$ Dipartimento di Fisica, Università di Genova, Via Dodecaneso 33, 16146 Genova, Italy \\ ${ }^{2}$ CNR-SPIN, Via Dodecaneso 33, 16146 Genova, Italy \\ ${ }^{3}$ Aix Marseille Université, Université de Toulon, CNRS, CPT, Marseille, France \\ ${ }^{4}$ CAMD, Department of Physics, Technical University of Denmark, $2800 \mathrm{Kgs}$. Lyngby, Denmark \\ ${ }^{5}$ Istituto Italiano di Tecnologia, Graphene Labs, Via Morego 30, I-16163 Genova, Italy
}

(Received 20 November 2018; revised manuscript received 7 February 2019; published 6 May 2019)

\begin{abstract}
We investigate heat current fluctuations induced by a periodic train of Lorentzian-shaped pulses, carrying an integer number of electronic charges, in a Hong-Ou-Mandel (HOM) interferometer implemented in a quantum Hall bar in the Laughlin sequence. We demonstrate that the noise in this collisional experiment cannot be reproduced in a setup with an effective single drive, in contrast to what is observed in the charge noise case. Nevertheless, the simultaneous collision of two identical levitons always leads to a total suppression even for the HOM heat noise at all filling factors, despite the presence of emergent anyonic quasiparticle excitations in the fractional regime. Interestingly, the strong correlations characterizing the fractional phase are responsible for a remarkable oscillating pattern in the HOM heat noise, which is completely absent in the integer case. These oscillations may be related to the recently predicted crystallization of levitons in the fractional quantum Hall regime.
\end{abstract}

DOI: 10.1103/PhysRevB.99.205406

\section{INTRODUCTION}

The recent progress in generating and controlling coherent few-particle excitations in quantum conductors paved the way for a new research field, known as electron quantum optics (EQO) $[1,2]$. The main purpose of EQO is to reconsider conventional optics experiments in the realm of condensedmatter physics, where propagating electronic wave packets are used instead of photons traveling along waveguides. In addition to the intrinsic interest in exploring the effect of Fermi statistics on traditional optical setups, EQO brings into play new physics linked to electron-electron interactions that is totally absent in the context of photonic quantum optics.

In this context, a remarkable effort has been put forth by the condensed-matter community to implement on-demand sources of electronic wave packets in mesoscopic systems. After seminal theoretical works and groundbreaking experimental results, two main methods to realize single-electron sources assumed a prominent role in the field of EQO [3-7]. The first injection protocol relies on the periodic driving of the discrete energy spectrum of a quantum dot, which plays the role of a mesoscopic capacitor [8-10]. In this way, it is possible to achieve the periodic injection of an electron and a hole along the ballistic channels of a system coupled to this mesoscopic capacitor through a quantum point contact (QPC) [11-14].

A second major step has been the recent realization of an on-demand source of electrons through the application of a time-dependent voltage to a quantum conductor [5,6,15-19].

*ronetti@fisica.unige.it
The main challenge to face, in this case, has been that an ac voltage would generally excite unwanted neutral electronhole pairs, thus spoiling at its heart the idea of a singleelectron source. The turning point to overcome this issue was the theoretical prediction by Levitov and co-workers that a periodic train of quantized Lorentzian-shaped pulses, carrying an integer number of particles per period, is able to inject minimal single-electron excitations devoid of any additional electron-hole pair, then termed levitons [20-22]. Indeed, this kind of single-electron source is simple to realize and operate, since it relies on usual electronic components, and potentially provides a high level of miniaturization and scalability. For their fascinating properties [23], levitons have been proposed as flying qubits [24] and as a source of entanglement [25-28] with appealing applications for quantum-information processing. Moreover, quantum tomography protocols able to reconstruct their single-electron wave functions have been proposed [29-31] and experimentally realized [32].

While the implementation of single-electron sources has not been a trivial task, the condensed-matter analogs of other quantum optics experimental components can be found in a more natural way. The waveguides for photons can be replaced by the ballistic edge channels of mesoscopic devices, such as quantum Hall systems. Moreover, the role of electronic beam splitter, which should mimic the half-silvered mirror of conventional optics, can be played by a QPC, where electrons are reflected or transmitted with a tunable probability, which is typically assumed as energy-independent. By combining these elements with the single-electron sources previously described, interferometric setups, originally conceived for optics experiments, can be implemented also in the condensed-matter realm $[33,34]$. One famous example is 
the Hanbury-Brown-Twiss (HBT) interferometer [35], where a stream of electronic wave packets is excited along ballistic channels and partitioned against a QPC [12]. The shot noise signal, generated due to the granular nature of electrons [36,37], was employed to probe the single-electron nature of levitons in a noninteracting two-dimensional electron gas $[15,38]$. Its extension to the fractional quantum Hall regime was considered in Ref. [39], where it was shown that levitons are minimal excitations also in strongly correlated edge channels.

A fundamental achievement of EQO has been the implementation of the Hong-Ou-Mandel (HOM) interferometer [40], where electrons impinge on the opposite side of a QPC with a tunable delay $[6,38,41]$. By performing this kind of collisional experiment, it is possible to gather information about the forms of the impinging electronic wave packets and to measure their degree of indistinguishability by accessing the zero-frequency noise $[14,16,42]$, namely without resorting to more complicated time-resolved, i.e., high-frequency measurements. For instance, when two indistinguishable and coherent electronic states collide simultaneously (zero time delay) at the QPC, charge current fluctuations are known to vanish at zero temperature, thus showing the so called Pauli dip $[6,38,43]$. This dip can be interpreted in terms of antibunching effects related to the Fermi statistics of electrons. HOM experiments can thus be employed to test whether decoherence and dephasing, induced by electronelectron interactions, reduce the degree of indistinguishability of colliding electrons [31,44-48].

As discussed above, the main driving force behind EQO has been to properly revise quantum optics experiments focusing on charge transport properties of single-electron excitations. Nevertheless, some recent groundbreaking experiments have spurred the investigation also in the direction of heat transport at the nanoscale [49-54]. In this context, the coherent transport and manipulation of heat fluxes have been reported in Josephson junctions [55-57] and quantum Hall systems [58-60]. Intriguingly, the quantization of heat conductance has been observed in integer [61] and fractional quantum Hall systems [62-64], which were already known for the extremely precise quantization of their charge conductance. In this way, ample and valuable information about these peculiar states of matter, which was not accessible by charge measurement, is now available with interesting implications also for quantum computation [65-68]. New intriguing challenges posed by extending concepts such as energy harvesting [69-75], driven heat and energy transport [76-80], energy exchange in open systems $[81,82]$, and fluctuation-dissipation theorems [83-86] to the quantum realm resulted in a great deal of progress in the field of quantum thermodynamics.

A new perspective on EQO has also been triggered by the rising interest in heat transport properties of single-electron excitations. For instance, heat current was revealed as a useful resource for the full reconstruction of a single-electron wave function [87]. Intriguingly, also fluctuations of heat transport properties, such as mixed-charge correlators [88-90] and heat current noise [91,92], were investigated in the case of single-electron sources, and, in particular, it was shown that levitons are minimal excitations also for heat transport [93]. Even though an experimental observation of heat current

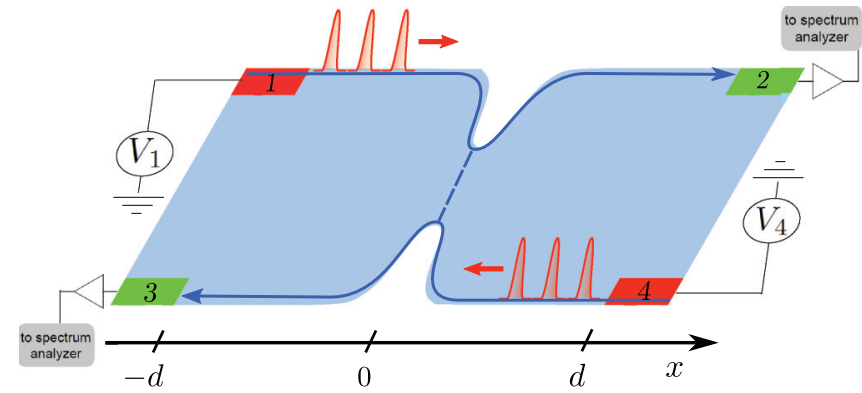

FIG. 1. Four-terminal setup for Hong-Ou-Mandel interferometry in the FQH regime. Contacts 1 and 4 are used as input terminals, while contacts 2 and 3 are the output terminals. The latter are connected to detectors where current and noise are measured.

fluctuations is still lacking, an experimental protocol has been recently proposed in order to access this quantity through temperature fluctuations [94].

Motivated by these recent developments, we address the problem of the heat noise generated by levitons injected in a HOM interferometer in the fractional quantum Hall regime. We consider a four-terminal quantum Hall bar in the Laughlin sequence [95], where a single channel arises on each edge. Two terminals are contacted to time-dependent voltages, namely $V_{1}$ and $V_{4}$. Tunneling processes of quasiparticles are allowed by the presence of a QPC connecting the two edge states. In this case, charge noise generated in the HOM setup is identical to the one generated in an effective single-drive setup driven by the voltage $V_{1}-V_{4}$. Interestingly, we prove that this does not hold true anymore for heat noise, since it is possible to identify a contribution to HOM heat noise, which is absent in an interferometer driven by the effective single-drive $V_{1}-V_{4}$. In addition, we prove that the HOM heat noise always vanishes for a zero delay between the driving voltage, both for integer and fractional filling factors. Finally, we focus on the case of Lorentzian-shaped voltage carrying an integer number of electrons, and we show that the HOM heat noise displays unexpected side dips in the fractional quantum Hall regime, which have no parallel in the integer regime. Intriguingly, the number of these side dips increases with the number of levitons injected per period. This result is consistent with the recently predicted phenomenon of charge crystallization of levitons in the fractional quantum Hall regime [96].

The paper is organized as follows. In Sec. II, we introduce the model and the setup. Then, we evaluate charge and heat noises in Sec. III. In Sec. IV, we present our results focusing on the peculiar case of levitons. Finally, we draw the conclusions in Sec. V. Three Appendixes are devoted to the technical aspects.

\section{MODEL}

A quantum Hall bar in a four-terminal geometry is depicted in Fig. 1. In the Laughlin sequence $v=\frac{1}{2 n+1}$, with integer $n \geqslant 0$, a single chiral mode arises on each edge [95,97]. In the special case of the integer quantum Hall effect at $v=1(n=0)$, the system is composed of ordinary fermions and the chiral edge states are one-dimensional Fermi liquids. 
This description fails for other filling factors, where the excitations are quasiparticles with fractional charge $-v e$ (with $e>$ 0 ). The low-energy properties of the Laughlin states are well captured by a hydrodynamical model formulated in terms of right-moving and left-moving bosonic edge modes $\Phi_{R / L}(x)$, which satisfy commutation relations $\left[\Phi_{R / L}(x), \Phi_{R / L}(y)\right]=$ $\pm i \pi \operatorname{sgn}(x-y)$. The free Hamiltonian of these edge modes is (we set $\hbar=1$ throughout the paper) [98]

$$
H_{0}=\frac{v}{4 \pi} \int d x \sum_{r=R, L}\left[\partial_{x} \Phi_{r}(x)\right]^{2},
$$

where $v$ is the velocity of propagation of right- and leftmoving bosonic modes.

Terminals 1 and 4 are assumed to be connected to external time-dependent drives, while the remaining terminals are used to perform measurements. The charge densities, defined as

$$
\rho_{R / L}(x)= \pm \frac{e \sqrt{v}}{2 \pi} \partial_{x} \Phi_{R / L}(x),
$$

are capacitively coupled to the gate potentials $\mathcal{V}_{1 / 4}(x, t)$ through the following gate Hamiltonian [99-101]:

$$
H_{g}=\int d x\left\{\mathcal{V}_{1}(x, t) \rho_{R}(x)+\mathcal{V}_{4}(x, t) \rho_{L}(x)\right\} .
$$

The spatial dependence of the potentials is restricted to the region containing the semi-infinite contacts $1(R)$ and $4(L)$ by putting $\mathcal{V}_{1}(x, t)=\Theta(-(x+d)) V_{1}(t)$ and $\mathcal{V}_{4}(x, t)=\Theta(x-$ d) $V_{4}(t)$ (with $d>0$ ). Here, $V_{1 / 4}(t)=V_{1 / 4, \mathrm{dc}}+V_{1 / 4, \mathrm{ac}}(t)$ are periodic voltages, where $V_{1 / 4 \text {,dc }}$ are time-independent dc components and $V_{1 / 4 \text {,ac }}$ are pure periodic ac signals with period $\mathcal{T}=\frac{2 \pi}{\omega}$, such that $\int_{0}^{\mathcal{T}} \frac{d t}{\mathcal{T}} V_{1 / 4}(t)=V_{1 / 4, \mathrm{dc}}$. We remark that such modelization of the electromagnetic coupling between gate voltages and Hall bar occurs for gauge fixing with zero vector potential.

Since backscattering between the two edges is exponentially suppressed, we introduce a quantum point contact (QPC) at $x=0$, as shown in Fig. 1, in order to allow for tunneling events between right- and left-moving excitations. The assumption of a pointlike, i.e., energy-independent, scatterer is motivated by realistic experiments, where additional nonlinearities are usually avoided $[38,41]$, and by their excellent agreement with an energy-independent scattering matrix approach [3]. We suppose that the QPC is tuned to a very low reflectivity, i.e., in the weak backscattering regime, where the tunneling of fractional quasiparticles is the only relevant process [102-104]. The corresponding additional term in the Hamiltonian is

$$
H_{t}=\Lambda \Psi_{R}^{\dagger}(0) \Psi_{L}(0)+\text { H.c., }
$$

where we introduced the quasiparticle fields represented by the bosonization identity $[36,105,106]$

$$
\Psi_{R / L}(x)=\frac{\mathcal{F}_{R / L}}{\sqrt{2 \pi a}} e^{-i \sqrt{v} \Phi_{R / L}(x)},
$$

with $\mathcal{F}_{R / L}$ the so-called Klein factor, necessary for the proper anticommutation relations, and $a$ is the short-length cutoff.

\section{NOISES IN THE DOUBLE-DRIVE CONFIGURATION}

The random partitioning, due to the Poissonian tunneling at the QPC, generates fluctuations in the currents flowing along the quantum Hall bar. In this section, we derive the expressions for charge and heat current noise in the double-drive configuration introduced in Sec. II, focusing on the regions downstream of the voltage contacts, namely $-d \leqslant x \leqslant d$.

\section{A. Charge noise}

We start by recalling the calculations for charge noise $[3,15,39]$. Charge current operators entering reservoirs 2 and 3 (located in $x=-d$ and $x=d$, respectively) can be expressed, due to chirality of Laughlin edge states, in terms of charge densities in Eq. (2),

$$
j_{2 / 3}(t)= \pm v \rho_{R / L}( \pm d, t) .
$$

The zero-frequency cross-correlated charge noise is

$$
\mathcal{S}_{C}=\int_{0}^{\mathcal{T}} \frac{d t}{\mathcal{T}} \int_{-\infty}^{+\infty} d t^{\prime}\left[\left\langle j_{2}\left(t^{\prime}\right) j_{3}(t)\right\rangle-\left\langle j_{2}\left(t^{\prime}\right)\right\rangle\left\langle j_{3}(t)\right\rangle\right],
$$

where the thermal average is performed over the initial equilibrium density matrix, in the absence of tunneling and driving voltage. In the weak backscattering regime, the standard perturbative approach in the tunneling Hamiltonian will be used. The total time evolution of charge current operators with respect to $H_{0}+H_{g}+H_{t}$ can then be constructed in terms of powers of $\Lambda$ and reads

$$
j_{2 / 3}(t)=j_{2 / 3}^{(0)}(t)+j_{2 / 3}^{(1)}(t)+j_{2 / 3}^{(2)}(t)+O\left(|\Lambda|^{3}\right),
$$

with

$$
\begin{gathered}
j_{2 / 3}^{(0)}(t)= \pm v \rho_{R / L}^{(0)}( \pm d, t), \\
j_{2 / 3}^{(1)}(t)= \pm i v \int_{-\infty}^{t} d t^{\prime}\left[H_{t}\left(t^{\prime}\right), \rho_{R / L}^{(0)}( \pm d, t)\right], \\
j_{2 / 3}^{(2)}(t) \\
= \pm(i)^{2} v \int_{-\infty}^{t} d t^{\prime} \int_{-\infty}^{t^{\prime}} d t^{\prime \prime}\left[H_{t}\left(t^{\prime \prime}\right),\left[H_{t}\left(t^{\prime}\right), \rho_{R / L}^{(0)}( \pm d, t)\right]\right]
\end{gathered}
$$

where the tunneling Hamiltonian $H_{t}(t)$ and the charge densities $\rho_{R / L}^{(0)}(x, t)$ evolve in the interaction picture with respect to $H_{0}+H_{g}$. To make explicit the form of $\rho_{R / L}^{(0)}(x, t)$, it is sufficient to solve the equations of motion for the bosonic fields $\Phi_{R / L}$ with respect to $H_{0}+H_{g}$, i.e., in the absence of tunneling. The solutions read

$$
\Phi_{R / L}(x, t)=\phi_{R / L}(x, t)-e \sqrt{v} \int_{0}^{t \mp \frac{x}{v}-\frac{d}{v}} d s V_{1 / 4}(s),
$$

where $\phi_{R / L}(x, t)=\phi_{R / L}(x \mp v t)$ are the chiral bosonic fields at equilibrium (zero applied drive).

By exploiting the commutator

$$
\left[H_{t}\left(t^{\prime}\right), \rho_{R / L}^{(0)}(x, t)\right]=-\delta\left(t^{\prime}-\left(t \mp \frac{x}{v}\right)\right) \dot{N}_{R / L}(x, t),
$$


where

$$
\begin{gathered}
\dot{N}_{R}(x, t)=i v e \Lambda \Psi_{R}^{\dagger}(x-v t, 0) \Psi_{L}(x-v t, 0)+\text { H.c. }, \\
\dot{N}_{L}(x, t)=-i v e \Lambda \Psi_{R}^{\dagger}(x+v t, 0) \Psi_{L}(x+v t, 0)+\text { H.c. }
\end{gathered}
$$

Equations (10) and (11) can be further recast as

$$
\begin{gathered}
j_{2 / 3}^{(1)}(t)=\dot{N}_{R / L}( \pm d, t), \\
j_{2 / 3}^{(2)}(t)=i \int_{-\infty}^{t-\frac{d}{v}} d t^{\prime \prime}\left[H_{t}\left(t^{\prime \prime}\right), \dot{N}_{R / L}( \pm d, t)\right] .
\end{gathered}
$$

In these expressions, we introduced the time evolution of quasiparticle fields with respect to $H_{0}+H_{g}$, which can be obtained from Eq. (12) using the bosonization identity

$$
\Psi_{R, L}(x, t)=\frac{\mathcal{F}_{R / L}}{\sqrt{2 \pi a}} e^{-i \sqrt{v} \phi_{R / L}(x, t)} e^{i v e \int_{0}^{t+\frac{x}{v}-\frac{d}{v}} d t^{\prime} V_{1 / 4}\left(t^{\prime}\right)} .
$$

The current noise can be obtained from Eqs. (9) and (10): the only nonvanishing contribution to second order in $\Lambda$ comes from $j_{2}^{(1)}(t+\tau) j_{3}^{(1)}(t)$, with terms $j_{2}^{(0)}(t+\tau) j_{3}^{(2)}(t)$ and $j_{2}^{(2)}(t+\tau) j_{3}^{(0)}(t)$ averaging to zero.

By introducing the correlator $\left(k_{B}=1\right)$

$$
\begin{aligned}
P_{2 v}\left(t^{\prime}-t\right) & =\left(\left\langle e^{i \sqrt{v} \phi_{R / L}\left(0, t^{\prime}\right)} e^{-i \sqrt{v} \phi_{R / L}(0, t)}\right\rangle\right)^{2} \\
& =\left[\frac{\pi \theta\left(t^{\prime}-t\right)}{\sinh \left[\pi \theta\left(t^{\prime}-t\right)\right]\left[1+i \omega_{c}\left(t^{\prime}-t\right)\right]}\right]^{2 v},
\end{aligned}
$$

with $\theta$ the temperature and $\omega_{c}=v / a$ the high-energy cutoff, one finds $\left(\lambda=\frac{\Lambda}{2 \pi a}\right)$

$$
\begin{aligned}
\mathcal{S}_{C}= & -2(v e)^{2}|\lambda|^{2} \int_{0}^{\mathcal{T}} \frac{d t}{\mathcal{T}} \int_{-\infty}^{+\infty} d t^{\prime} \\
& \times \cos \left\{v e \int_{t}^{t^{\prime}} V_{-}(\tau) d \tau\right\} P_{2 v}\left(t^{\prime}-t\right),
\end{aligned}
$$

where $V_{-}=V_{1}-V_{4}$.

Even though this charge noise is generated in a doubledrive configuration, it is interesting to point out that it actually depends only on the single effective drive $V_{-}(t)$. The configuration with a single drive is usually termed in the literature the Hanbury-Brown-Twiss (HBT) setup [12,35,38,107].

Therefore, the charge noise presented in Eq. (20) is the same as that generated in a single-drive configuration, where reservoir 4 is grounded $\left[V_{4}(t)=0\right]$ and reservoir 1 is contacted to the periodic voltage $V_{-}(t)$, such that

$$
S_{C}\left(V_{1}, V_{4}\right)=S_{C}\left(V_{-}, 0\right) \text {. }
$$

Here, the arguments in parentheses indicate the voltage applied to reservoirs 1 and 4, respectively.

One might consider Eq. (21) as a consequence of a trivial shift of both voltages by a value corresponding to $V_{4}$. Nevertheless, such a result cannot be obtained by means of a gauge transformation (see Appendix A). In this sense, Eq. (21) implies that the charge noise incidentally acquires the same expression in these two physically distinct experimental setups. As will be clearer in the following, for the charge case this is a consequence of the presence of a single local (energy-independent) QPC. Generally, we expect that the double-drive and the single-drive $\left[V_{1}(t)=V_{-}(t)\right.$ and $V_{4}(t)=$ $0]$ configurations return different outcomes for other physical observables, such as heat noise, as discussed in the next part.

\section{B. Heat noise}

In the following, we evaluate the correlation noise of heat current between terminals 2 and 3 in the double-drive configuration. The heat current operators of terminals 2 and 3 can be expressed in terms of heat density operators [108]

$$
\mathcal{Q}_{R / L}(x, t)=\frac{v}{4 \pi}\left[\partial_{x} \Phi_{R / L}(x, t)\right]^{2}
$$

as

$$
\mathcal{J}_{2 / 3}(t)= \pm v \mathcal{Q}_{R / L}( \pm d, t),
$$

due to the chirality of Laughlin edge states.

Then, we can define the cross-correlated heat noise as

$$
\mathcal{S}_{Q}=\int_{0}^{\mathcal{T}} \frac{d t}{\mathcal{T}} \int d t^{\prime}\left\{\left\langle\mathcal{J}_{2}\left(t^{\prime}\right) \mathcal{J}_{3}(t)\right\rangle-\left\langle\mathcal{J}_{2}\left(t^{\prime}\right)\right\rangle\left\langle\mathcal{J}_{3}(t)\right\rangle\right\}
$$

Analogously to charge current, one can expand heat current operators in power of the tunneling amplitude $\Lambda$, thus obtaining

$$
\mathcal{J}_{2 / 3}(t)=\mathcal{J}_{2 / 3}^{(0)}(t)+\mathcal{J}_{2 / 3}^{(1)}(t)+\mathcal{J}_{2 / 3}^{(2)}(t)+O\left(|\Lambda|^{3}\right),
$$

where

$$
\begin{gathered}
\mathcal{J}_{2 / 3}^{(0)}(t)= \pm v \mathcal{Q}_{R / L}^{(0)}( \pm d, t) \\
\mathcal{J}_{2 / 3}^{(1)}(t)= \pm i v \int_{-\infty}^{t} d t^{\prime}\left[H_{t}\left(t^{\prime}\right), \mathcal{Q}_{R / L}^{(0)}( \pm d, t)\right] \\
\mathcal{J}_{2 / 3}^{(2)}(t) \\
= \pm i^{2} v \int_{-\infty}^{t} d t^{\prime} \int_{-\infty}^{t^{\prime}} d t^{\prime \prime}\left[H_{t}\left(t^{\prime \prime}\right),\left[H_{t}\left(t^{\prime}\right), \mathcal{Q}_{R / L}^{(0)}( \pm d, t)\right]\right]
\end{gathered}
$$

In the above equations, we have denoted with $\mathcal{Q}^{(0)}(x, t)$ the time evolution of heat density in the absence of tunneling, which can be obtained from the time evolution of bosonic fields in Eq. (12), and it reads

$$
\begin{aligned}
\mathcal{Q}_{R / L}^{(0)}(x, t)= & \frac{v}{4 \pi}\left[\left(\partial_{x} \phi_{R / L}(x, t)\right)^{2}\right. \\
& \pm e \sqrt{v} \partial_{x} \phi_{R / L}(x, t) V_{1 / 4}\left(t \mp \frac{x}{v}\right) \\
& \left.+\frac{e^{2} v}{v} V_{1 / 4}^{2}\left(t \mp \frac{x}{v}\right)\right] .
\end{aligned}
$$

Let us notice that the expectation value at zero temperature for such an operator (i.e., the heat current generated in a translationally invariant edge state due to the application of a voltage bias) is given by

$$
\left\langle\mathcal{Q}_{R / L}^{(0)}(x, t)\right\rangle=\frac{e^{2} v}{4 \pi} V_{1 / 4}^{2}\left(t \mp \frac{x}{v}\right)=\frac{G_{0}}{2} V_{1 / 4}^{2}\left(t \mp \frac{x}{v}\right),
$$

where $G_{0}=v e^{2} /(2 \pi)$ is the Hall conductance ( $\hbar$ being set to $1)$. This term is quadratic in $V_{1 / 4}$, as one would indeed expect 
from Joule's law. A linear term in $V_{1 / 4}$ would actually correspond to a thermoelectric generation of heat into the topological edge state (as reported, for instance, in Refs. [109,110]), which is, however, not the case for the present model. This means that the flow of heat current cannot be reversed by reversing the sign of $V_{1 / 4}$, i.e., heat will always flow from the contact to the QPC.

The commutator

$$
\left[H_{t}\left(t^{\prime}\right), \mathcal{Q}_{R / L}^{(0)}(x, t)\right]=-i \delta\left(t^{\prime}-\left(t \mp \frac{x}{v}\right)\right) \dot{Q}_{R / L}(x, t),
$$

where

$$
\begin{array}{r}
\dot{Q}_{R}(x, t)=v \Lambda\left(\partial_{x} \Psi_{R}^{\dagger}(x, t)\right) \Psi_{L}(x, t)+\text { H.c. }, \\
\dot{Q}_{L}(x, t)=-v \Lambda \Psi_{R}^{\dagger}(x, t)\left[\partial_{x} \Psi_{L}(x, t)\right]+\text { H.c. }
\end{array}
$$

can be used to recast Eqs. (27) and (28),

$$
\begin{gathered}
\mathcal{J}_{2 / 3}^{(1)}(t)= \pm \dot{Q}_{R / L}( \pm d, t) \\
\mathcal{J}_{2 / 3}^{(2)}(t)= \pm i \int_{-\infty}^{t-\frac{d}{v}} d t^{\prime \prime}\left[H_{t}\left(t^{\prime \prime}\right), \dot{Q}_{R / L}( \pm d, t)\right] .
\end{gathered}
$$

The perturbative expansion of the heat current operator in Eq. (25) allows us to express heat correlation noise to lowest order as

$$
\mathcal{S}_{Q}=\mathcal{S}_{Q}^{(02)}+\mathcal{S}_{Q}^{(20)}+\mathcal{S}_{Q}^{(11)}+O\left(|\Lambda|^{3}\right),
$$

where

$\mathcal{S}_{Q}^{(i j)}=\int_{0}^{\mathcal{T}} \frac{d t}{\mathcal{T}} \int d t^{\prime}\left\{\left\langle\mathcal{J}_{2}^{(i)}\left(t^{\prime}\right) \mathcal{J}_{3}^{(j)}(t)\right\rangle-\left\langle\mathcal{J}_{2}^{(i)}\left(t^{\prime}\right)\right\rangle\left\langle\mathcal{J}_{3}^{(j)}(t)\right\rangle\right\}$.

Now, we can perform standard calculations, whose details are given in Appendix B, in order to evaluate all the terms appearing in Eq. (36). By using the result of this calculation, it is possible to check whether an expression analogous to Eq. (21) holds true also for heat noise. Interestingly, one finds that

$$
\mathcal{S}_{Q}\left(V_{1}, V_{4}\right)=\mathcal{S}_{Q}\left(V_{-}, 0\right)+\Delta \mathcal{S}_{Q}\left(V_{1}, V_{4}\right),
$$

thus showing that, in contrast with the charge sector, heat fluctuations generated in the double-drive or in the singledrive configurations are different. The two contributions in Eq. (38) are

$$
\begin{aligned}
& \mathcal{S}_{Q}\left(V_{-}, 0\right) \\
& =|\lambda|^{2} \int_{0}^{\mathcal{T}} \frac{d t}{\mathcal{T}} \int d t^{\prime}\left\{\cos \left(v e \int_{t}^{t^{\prime}} d \tau V_{-}(\tau)\right)\right. \\
& \quad \times \operatorname{Re}\left[\mathcal{P}_{\nu}\left(t^{\prime}-t\right) \partial_{t}^{2} \mathcal{P}_{\nu}\left(t^{\prime}-t\right)\right] \\
& \quad+\frac{v e v}{\pi} \int d t^{\prime \prime} V_{-}\left(t^{\prime}\right) \mathcal{K}\left(t^{\prime}, t, t^{\prime \prime}\right) \sin \left(v e \int_{t}^{t^{\prime}} d \tau V_{-}(\tau)\right) \\
& \left.\quad \times \operatorname{Im}\left[\partial_{t^{\prime \prime}} \mathcal{P}_{2 v}\left(t^{\prime \prime}-t\right)\right]\right\},
\end{aligned}
$$

$$
\begin{aligned}
\Delta \mathcal{S}_{Q}\left(V_{1}, V_{4}\right)= & v^{2} e^{2}|\lambda|^{2} \int_{0}^{\mathcal{T}} \frac{d t}{\mathcal{T}} \int d t^{\prime} \cos \left(v e \int_{t}^{t^{\prime}} d \tau V_{-}(\tau)\right) \\
& \times\left(\alpha_{R L}\left(t, t^{\prime}\right) \operatorname{Re}\left[\mathcal{P}_{2 v}\left(t^{\prime}-t\right)\right]+\beta_{R L}\left(t, t^{\prime}\right)\right. \\
& \left.\times \operatorname{Im}\left[\mathcal{P}_{2 v}\left(t^{\prime}-t\right)\right]\right),
\end{aligned}
$$

where we defined the following functions:

$$
\begin{gathered}
\mathcal{K}\left(t^{\prime}, t, t^{\prime \prime}\right)=\int d \tau \mathcal{P}_{2}\left(t^{\prime}-\tau\right)\left[\Theta\left(\tau-t^{\prime \prime}\right)-\Theta(\tau-t)\right] \\
=\frac{\pi \theta}{v} \frac{\sinh \left[\pi \theta\left(t-t^{\prime \prime}\right)\right]}{\sinh \left[\pi \theta\left(t^{\prime}-t\right)\right] \sinh \left[\pi \theta\left(t^{\prime}-t^{\prime \prime}\right)\right]}, \\
\alpha_{R L}\left(t, t^{\prime}\right)=\left[V_{1}(t) V_{4}\left(t^{\prime}\right)-V_{4}(t) V_{1}\left(t^{\prime}\right)\right], \\
\beta_{R L}=\frac{v}{\pi} \int d t^{\prime \prime} \mathcal{K}\left(t^{\prime \prime}, t, t^{\prime}\right) V_{1}\left(t^{\prime \prime}\right)\left[V_{4}\left(t^{\prime}\right)-V_{4}(t)\right] .
\end{gathered}
$$

The result of Eq. (38) arises because heat noise is sensitive to the energy distribution of the injected particles, thus leading to different outcomes in the single- and double-drive configurations. In this light, we expect this to hold true for general energy-dependent phenomena occurring at the QPC. For instance, any similarity between charge noises generated in the two setups discussed previously would disappear for more complicated tunneling geometry, such as multiple QPC or extended contacts, where transmission functions become energy-dependent [109-113]. Nevertheless, the presence of an extended scattering region would modify at its core the concept of a HOM interferometer, since the simultaneous collision of identical incoming particles cannot be guaranteed anymore. Consequently, even though charge current noise due to an energy-dependent scatterer is expected to share some similarities with heat current noise in our setup, it is only the latter quantity that can be interpreted as generated in a proper HOM interferometer.

Equation (38) further indicates that the double-drive and the single-drive configurations are completely distinct setups and that the relation in Eq. (21) is solely a contingent effect of the single local QPC geometry. It is useful to express heat correlation noise in energy space by introducing the following Fourier series:

$$
\begin{aligned}
\nu e V_{1 / 4}(t) & =\sum_{k} c_{k, R / L} e^{i k \omega t}, \\
e^{-i v e \int_{0}^{t} d \tau V_{-}(\tau)} & =\sum_{l} \tilde{p_{l}} e^{-i\left(l+q_{1}-q_{4}\right) \omega t},
\end{aligned}
$$

where we defined also the number of particles excited by $V_{1 / 4}$ along the system in a period

$$
q_{1 / 4}=\frac{v e}{2 \pi} \int_{0}^{\mathcal{T}} d t V_{1 / 4}(t)=\frac{v e V_{1 / 4, d c}}{\omega},
$$

and the Fourier transform of $\mathcal{P}_{g}(t)$ in Eq. (19)

$$
\begin{aligned}
\tilde{\mathcal{P}}_{g}(E) & =\int d t \mathcal{P}_{g}(t) e^{i E t} \\
& =\left(\frac{2 \pi \theta}{\omega_{c}}\right)^{g-1} \frac{e^{\frac{E}{2 \theta}}}{\Gamma(g) \omega_{c}}\left|\Gamma\left(\frac{g}{2}-i \frac{E}{2 \pi \theta}\right)\right|^{2} .
\end{aligned}
$$


By exploiting these results, the two contributions to $\mathcal{S}_{Q}$ become

$$
\begin{gathered}
\mathcal{S}_{Q}\left(V_{-}, 0\right)=-|\lambda|^{2} \sum_{l}\left\{\frac{2 v^{2} \pi^{2} \theta^{2}+(1+v)\left[\left(l+q_{1}-q_{4}\right) \omega\right]^{2}}{1+2 v}\left|\tilde{p}_{l}\right|^{2} \tilde{\mathcal{P}}_{2 v}\left[\left(l+q_{1}-q_{4}\right) \omega\right]\right. \\
\left.-\frac{1}{4} \sum_{k \neq 0}\left(c_{k, 1}-c_{k, 4}\right)\left(\tilde{p}_{l-k} \tilde{p}_{l}^{*}-\tilde{p}_{l} \tilde{p}_{l+k}^{*}\right)\left(l+q_{1}-q_{4}\right) \omega \operatorname{coth} \frac{k \omega}{2 \theta}\left(\tilde{\mathcal{P}}_{2 v}\left[\left(l+q_{1}-q_{4}\right) \omega\right]-\tilde{\mathcal{P}}_{2 v}\left[-\left(l+q_{1}-q_{4}\right) \omega\right]\right)\right\} \\
\Delta \mathcal{S}_{Q}\left(V_{1}, V_{4}\right)=\frac{|\lambda|^{2}}{2} \sum_{k, p, l}\left(c_{k, 1} c_{p, 4}-c_{k, 4} c_{p, 1}\right) \tilde{p}_{l+k+p} \tilde{p}_{l}^{*} \frac{\mathcal{W}_{\left(l+q_{1}-q_{4}\right), k, p}+\mathcal{W}_{\left(l+q_{1}-q_{4}\right), p, k}}{2}
\end{gathered}
$$

where the coefficients $\mathcal{W}_{l, k, p}$ encode all the effects due to temperature and interaction on $\Delta \mathcal{S}_{Q}$ and read

$$
\begin{aligned}
\mathcal{W}_{l, k, p}= & \frac{\omega_{c}}{4 \pi} \int \frac{d E}{2 \pi}\left\{\tilde{\mathcal{P}}_{1}(E) \tilde{\mathcal{P}}_{1}(k \omega-E)\left[\tilde{\mathcal{P}}_{2 v-1}(E-l \omega)+\tilde{\mathcal{P}}_{2 v-1}[-E-(l+k+p) \omega]+\tilde{\mathcal{P}}_{2 v-1}(-E+l \omega)\right]\right. \\
& \left.+\tilde{\mathcal{P}}_{2 v-1}[E+(l+k+p) \omega]\right\}-\frac{1}{2}\left(\tilde{\mathcal{P}}_{2 v}[(l+k) \omega]+\tilde{\mathcal{P}}_{2 v}[-(l+k)]\right) .
\end{aligned}
$$

Let us observe that the contribution $\Delta \mathcal{S}_{Q}$ exists only in the double-drive configurations. Indeed, in the configuration with a single drive, where $V_{4}=0$, one obtains that $c_{k, 4}=0$ for each $k$, and the contribution in Eq. (49) vanishes.

Before concluding this section, we also provide the expression of the HOM heat noise in the fermionic case at $v=1$. In this case, Eqs. (48) and (49) read

$$
\begin{aligned}
\mathcal{S}_{Q}\left(V_{-}, 0\right)=-|\lambda|^{2} \sum_{l} & \left\{\frac{2 \pi^{2} \theta^{2}+2\left[\left(l+q_{1}-q_{4}\right) \omega\right]^{2}}{3}\left|\tilde{p}_{l}\right|^{2} \tilde{\mathcal{P}}_{2}\left[\left(l+q_{1}-q_{4}\right) \omega\right]-\sum_{k \neq 0} c_{k,-} c_{-k,-} k \omega \operatorname{coth}\left(\frac{k \omega}{2 \theta}\right)\right\}, \\
\Delta \mathcal{S}_{Q}\left(V_{1}, V_{4}\right)= & \frac{|\lambda|^{2}}{4} \sum_{k}\left(c_{k,+} c_{-k,+}-c_{k,-} c_{-k,-}\right) \tilde{\mathcal{P}}_{2}(k \omega) \\
& -\sum_{k, q, l}\left(c_{k,+} c_{q,+}-c_{k,-} c_{q,-}\right) \tilde{p}_{l+k+q} \tilde{p}_{l-k}^{*}\left(\tilde{\mathcal{P}}_{2}\left[\left(l+q_{1}-q_{4}\right) \omega\right]+\tilde{\mathcal{P}}_{2}\left[-\left(l+q_{1}-q_{4}\right) \omega\right]\right) .
\end{aligned}
$$

We observe that the two terms appearing in Eq. (51) correspond to the ones evaluated in Ref. [92] resorting to the Floquet scattering matrix formalism in the presence of a single external drive. In this case, the two terms can be respectively linked to transport of particles between different reservoirs and to scattering between different energy states in the same reservoir [90,92]. According to these considerations, the first term in Eq. (48) can be easily identified as quasiparticle transport across the sample for a generic filling factors, due to the similarity with the transport contribution in Eq. (51). Nevertheless, the second term in Eq. (48) displays a much more complex structure in the presence of a fractional filling factor, thus complicating its interpretation as a simple reservoir contribution.

\section{Hong-Ou-Mandel noises}

Among all the possible choices for the configuration involving the two voltages $V_{1}$ and $V_{4}$, one of the most interesting, even from the experimental point of view, is the Hong-OuMandel (HOM) setup, where two identical voltage drives are applied to reservoirs 1 and 4 and delayed by a constant time $t_{D}$. This experimental configuration corresponds to set $V_{1}(t)=V(t)$ and $V_{4}(t)=V\left(t+t_{D}\right)$ in Eq. (20), with $V(t)$ a generic periodic drive. In this situation the charges excited by each drive along the edge channels are equal, such that $q_{1}=q_{4}=q$.

For notational convenience, we define the single-drive heat noise and the HOM charge and heat noises as

$$
\begin{gathered}
\mathcal{S}_{Q}^{\mathrm{sd}}=\mathcal{S}_{Q}\left(V_{-}(t), 0\right), \\
\mathcal{S}_{C / Q}^{\mathrm{HOM}}=\mathcal{S}_{C / Q}\left(V(t), V\left(t+t_{D}\right)\right) .
\end{gathered}
$$

Let us notice that in the equations above, $\mathcal{S}_{Q}^{\text {sd }}$ represents the heat noise generated when the voltage $V_{-}(t)=V_{1}(t)-V_{4}(t)$ is applied to terminal 1 , while $\mathcal{S}_{C / Q}^{\mathrm{HOM}}$ are charge and heat noises when both terminals 1 and 4 are driven by $V_{1}(t)=$ $V(t)$ and $V_{4}=V\left(t+t_{D}\right)$, respectively. According to Eq. (38) and using the above definitions, the HOM heat noise can be expressed as

$$
\mathcal{S}_{Q}^{\mathrm{HOM}}=\mathcal{S}_{Q}^{\mathrm{sd}}+\Delta \mathcal{S}_{Q}
$$

From the existing literature $[3,39,96]$, it is well established that charge HOM noise reduces to its equilibrium value for null time delay. Before entering into the details of our discussion, we would like to prove analytically that the same holds true for HOM heat noise $\mathcal{S}_{Q}^{\mathrm{HOM}}$, independently of the choice of any parameter. The photoassisted amplitude in Eq. (45) reduces to $\tilde{p_{l}}=\delta_{l, 0}$ and the Fourier coefficients $c_{k,-}$ vanish for all $k$. Let us start by looking at the single-drive contribution. 
By substituting this analytical simplification in Eq. (48), we obtain

$$
\mathcal{S}_{Q}^{\mathrm{sd}}\left(t_{D}=0\right)=-|\lambda|^{2} \frac{\nu^{2} \pi^{2} \theta^{2}}{1+2 v} \equiv \mathcal{S}_{Q}^{\mathrm{vac}},
$$

which is independent of the injected particles and corresponds simply to the equilibrium noise $\mathcal{S}_{Q}^{\text {vac }}$ due to thermal fluctuations. This can be clearly understood given the fact that $V_{-}(t)=0$ for $t_{D}=0$ and the single-drive contribution corresponds to the noise generated in a driveless configuration.

Concerning the remaining part in Eq. (55), one has for $t_{D}=0$

$$
\Delta \mathcal{S}_{Q}=\frac{|\lambda|^{2}}{4} \sum_{k} c_{k, 1} c_{-k, 1}\left(\mathcal{W}_{0, k,-k}+\mathcal{W}_{0,-k, k}\right)
$$

where

$$
\mathcal{W}_{0, k,-k}=\frac{\tilde{\mathcal{P}}_{2 v}(k \omega)-\tilde{\mathcal{P}}_{2 v}(-k \omega)}{2} .
$$

From Eq. (58), we can clearly deduce that $\mathcal{W}_{0, k,-k}=$ $-\mathcal{W}_{0,-k, k}$, which enforces the vanishing of $\Delta \mathcal{S}_{Q}$ in Eq. (57). This is enough to prove that HOM heat noise always reaches its equilibrium value at $t_{D}=0$, such that

$$
\mathcal{S}_{Q}^{\mathrm{HOM}}\left(t_{D}=0\right)=\mathcal{S}_{Q}^{\mathrm{sd}}\left(t_{D}=0\right)=\mathcal{S}_{Q}^{\mathrm{vac}} .
$$

Let us note that this is not a trivial result since $\mathcal{S}_{Q}^{\mathrm{HOM}}$ does not depend effectively on the effective single drive $V_{-}$as $\mathcal{S}_{Q}^{\text {sd }}$, but on both $V_{1}$ and $V_{4}$, and even at $t_{D}=0$ the system is still driven by these two voltages.

\section{RESULTS AND DISCUSSIONS}

In this section, we discuss the results concerning the heat correlation noises in the HOM interferometer. In particular, we focus our discussion on a specific driving voltage, namely a periodic train of Lorentzian pulses

$$
V_{\text {Lor }}(t)=\frac{V_{0}}{\pi} \sum_{k=-\infty}^{+\infty} \frac{W}{W^{2}+(t-k \mathcal{T})^{2}} .
$$

A Lorentzian-shaped drive, which satisfy the additional quantization condition

$$
v e \int_{0}^{\mathcal{T}} d t V_{\mathrm{Lor}}(t)=2 \pi q,
$$

with $q$ an integer number, constitutes the optimal driving able to inject clean pulses devoid of any additional electron-hole pairs. The minimal excitations thus emitted into the quantum Hall channels are the aforementioned levitons $[20,22]$. The Fourier coefficients for this specific drive are given in Appendix C.

In the HOM setup previously described, a state composed by $q_{4}=q_{1}=q$ levitons [114] is injected by each driven contact and collide at the QPC, separated by a controllable time delay.

In analogy with the previous literature on charge noise, we introduce the following ratio $[30,39,44,46]$ :

$$
\mathcal{R}_{C / Q}^{\mathrm{HOM}}=\frac{\mathcal{S}_{C / Q}^{\mathrm{HOM}}-\mathcal{S}_{C / Q}^{\mathrm{vac}}}{2 \mathcal{S}_{C / Q}^{R}-2 \mathcal{S}_{C / Q}^{\mathrm{vac}}},
$$

where we subtracted the equilibrium noise $\mathcal{S}_{C / Q}^{\mathrm{vac}}$ and we normalize with respect to $\mathcal{S}_{C / Q}^{R} \equiv \mathcal{S}_{C / Q}\left(V_{1}, 0\right)$, which are charge and heat noises expected for the random partitioning of a single source of levitons, i.e., when $V_{1}(t)=V_{\mathrm{Lor}}(t)$ and $V_{4}(t)=$ 0 . The expressions for $\mathcal{S}_{C}^{\text {vac }}$ and $\mathcal{S}_{C}^{R}$ are well-known and have been derived in previous papers $[3,39,93]$. The expression for $\mathcal{S}_{Q}^{R}$ can be obtained from our results in Sec. III B and reads

$$
\begin{aligned}
\mathcal{S}_{Q}^{R}= & -|\lambda|^{2} \sum_{l}\left\{\frac{2 v^{2} \pi^{2} \theta^{2}+(1+v)(l \omega)^{2}}{1+2 v}\left|p_{l}\right|^{2} \tilde{\mathcal{P}}_{2 v}[(l+q) \omega]\right. \\
& -\sum_{k \neq 0} c_{k}\left(p_{l-k} p_{l}^{*}-p_{l} p_{l+k}^{*}\right)(l+q) \omega \frac{\tilde{\mathcal{P}}_{2}(k \omega)}{2 k \omega} \\
& \left.\times\left(\tilde{\mathcal{P}}_{2 v}[(l+q) \omega]-\tilde{\mathcal{P}}_{2 v}[-(l+q) \omega]\right)\right\}
\end{aligned}
$$

where $\quad c_{k}=v e \int_{0}^{\mathcal{T}} \frac{d t}{\mathcal{T}} V_{\mathrm{Lor}}(t) e^{i k \omega t}$ are the Fourier coefficients for a single Lorentzian voltage and $p_{l}=$ $\int_{0}^{\mathcal{T}} \frac{d t}{\mathcal{T}} e^{-i v e \int_{0}^{t} d \tau V_{\text {Lor }}(\tau)} e^{i(l+q) \omega t}$ (see Appendix C).

Let us notice that, according to Eq. (59), $\mathcal{R}_{Q}^{\mathrm{HOM}}$ vanishes for $t_{D}=0$. In the specific case of levitons, which are singleelectron excitations, at $v=1$ the physical explanation for the total dip at $t_{D}=0$ involves the antibunching effect of identical fermions: electronlike excitations colliding at the QPC at the same time are forced to escape on opposite channels, thus leading to a total suppression of fluctuations at $t_{D}=0$ and generating the so called Pauli dip [12,38,42]. For fractional filling factors, it is remarkable that this total dip is still present despite the presence of anyonic quasiparticles in the system, which do not obey Fermi-Pauli statistics [16,39]. Anyway, this single QPC geometry does not allow for the braiding of one quasiparticle around the other, thus excluding any possible effect due to fractional statistics.

In the following, we exploit the full generality of our derivation by performing the analysis for different values of $q$.

We start by considering the regime where thermal and quantum fluctuations are comparable. First, we focus on the relevant case of $q=1$, where states formed by a single leviton are injected from both sources [87]. The collision of identical single-leviton states is very interesting because previous work on fluctuations of charge current proved that in this case the ratio of HOM charge noise is independent of filling factors and temperatures, acquiring an universal analytical expression $[15,39]$. To perform a similar comparison for the heat noise, we present in Fig. 2 the HOM heat ratio considering two temperatures $\theta=0.25 \omega$ (solid line) and $\theta=0.5 \omega$ (dashed lines) for both the integer and the fractional case. Contrary to the charge case, these curves are all clearly distinct. This means that the universality in the analytical expression does not extend also to heat fluctuations. This fact can be explained by the dependence of heat HOM noise on the energy distribution of particles injected by the drives, which in turn is significantly affected by the temperature and by the strength of correlations encoded in the filling factor $v$. In particular, as the temperature is further increased, the thermal fluctuations tend to hide the effect of the voltages, resulting in a reduction of $\mathcal{R}_{Q}^{\mathrm{HOM}}$ for both filling factors. 

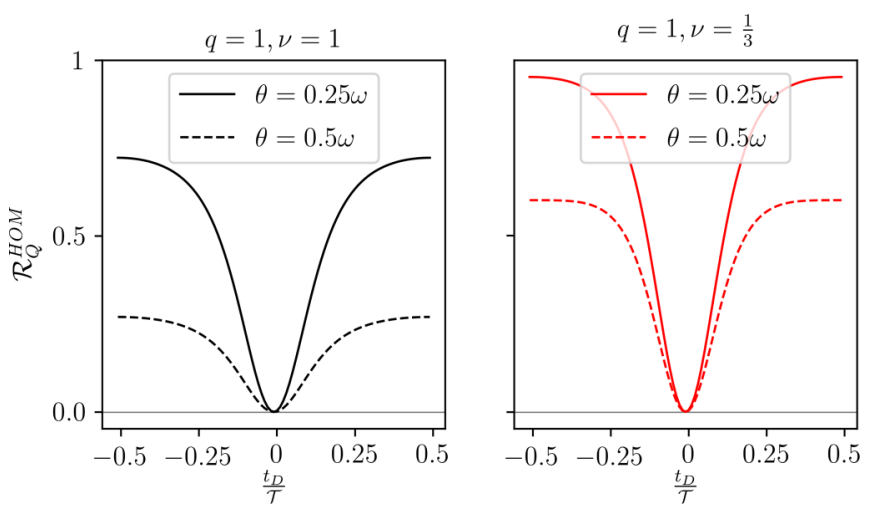

FIG. 2. HOM heat ratio $\mathcal{R}_{Q}^{\mathrm{HOM}}$ as a function of the time delay $t_{D}$ for $q=1$ and temperatures $\theta=0.25 \omega$ (solid lines) and $\theta=0.5 \omega$ (dashed lines). The integer case (left panel) and the fractional case for $v=\frac{1}{3}$ (right panel) are compared. The other parameters are $W=0.1 \mathcal{T}$ and $\omega=0.01 \omega_{c}$.

In Fig. 3, we start by looking at the collision of states composed of multiple levitons, and we compare HOM charge and heat ratios (solid and dashed lines, respectively) for $q=2$ and 4. In the fermionic case, presented in the two upper panels, both charge and heat ratio show a single smooth dip
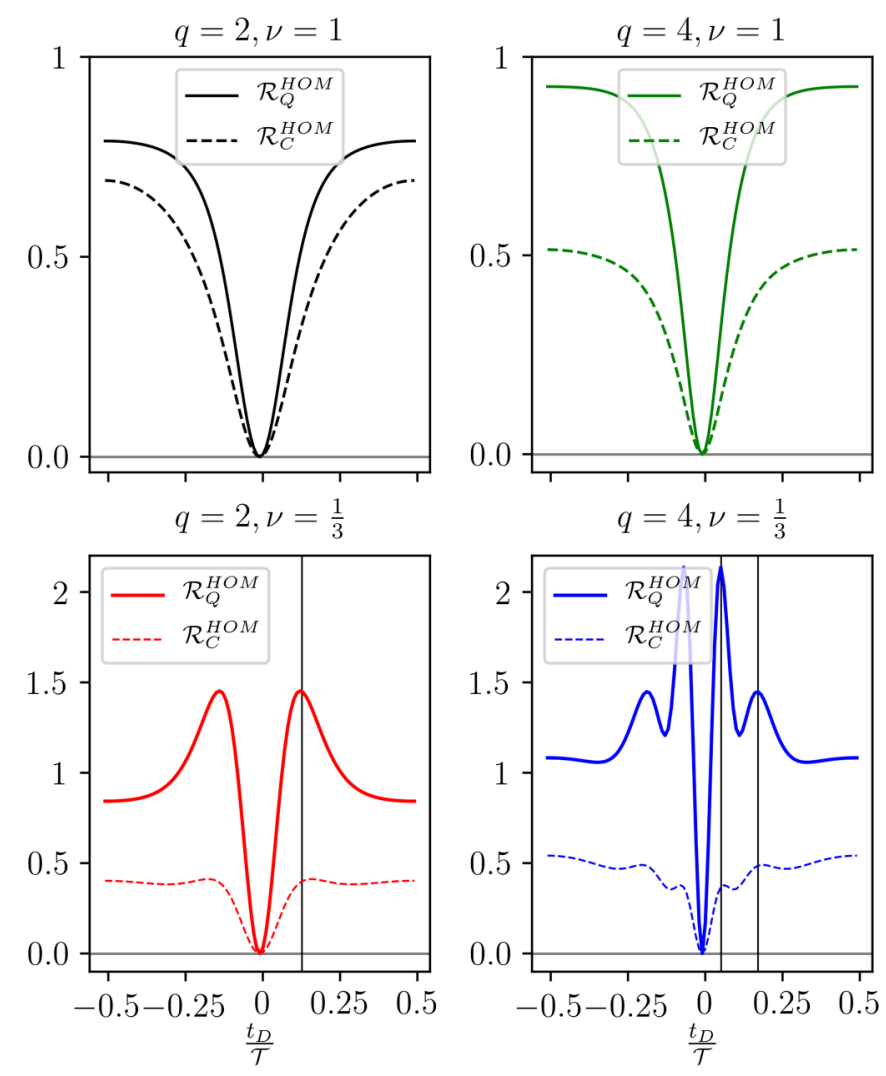

FIG. 3. HOM heat ratio $\mathcal{R}_{Q}^{\mathrm{HOM}}$ (solid lines) and HOM charge ratio $\mathcal{R}_{C}^{\mathrm{HOM}}$ (dashed lines) as a function of the time delay $t_{D}$ for $q=2$ and 4 . The integer case (upper panels) and the fractional case for $v=\frac{1}{3}$ (lower panels) are compared. Black vertical lines demonstrate the exact correspondence of side peaks appearing in a charge and heat ratio. The other parameters are $W=0.1 \mathcal{T}, \theta=0.25 \omega$, and $\omega=0.01 \omega_{c}$. at $t_{D}=0$, without additional side features. Interestingly, heat fluctuations are enhanced with respect to charge: in particular, heat HOM ratios saturate to their asymptotic value for smaller values of time delay compared to charge ratio. Again, the enhancement of heat fluctuations can be related to the fact that heat is not constrained by any conservation law, in contrast to the case of charge.

Very remarkably, the curves for the HOM ratio in the fractional case display instead some unexpected side peaks and dips in addition to the central dip. In particular, the number of these maxima and minima increases for states composed of more levitons. A recent paper by the authors explained this intriguing result for charge HOM noise in terms of a crystallization process induced by strong correlation on the charge density of $q$ levitons, i.e., a rearrangement of the density into an oscillating and ordered pattern with a number of peaks related to $q[96,115]$. Black vertical lines in the lower panel of Fig. 3 demonstrate the exact correspondence of side peaks appearing in charge and heat ratio as a function of time delay. Based on this argument, we can infer that the HOM heat noise is affected by the crystallization induced in the propagating levitons, thus giving rise to the features observed in the lower panel of Fig. 3. While the oscillating pattern of $\mathcal{R}_{Q}^{\mathrm{HOM}}$ remarkably matches with that of $\mathcal{R}_{C}^{\mathrm{HOM}}$, the amplitude oscillations are widely enhanced for heat fluctuations, in particular for the peaks occurring at small values of time delay.

We conclude by noticing that strong correlation of the fractional regime can increase the value of the HOM heat ratio even above 1 . Once again, since this is not the case for the single-drive contribution, this is due to the presence of $\Delta \mathcal{S}_{Q}$, which is peculiar to collision between levitons incoming from different reservoirs.

Now, we consider the regime of very low temperature $\theta \ll \omega$, where the quantum effects should be largely enhanced with respect to the thermal fluctuations. Having established from the previous discussion the connection between $\Delta \mathcal{S}_{Q}$ and $\mathcal{S}_{Q}^{\mathrm{HOM}}$ in the fractional regime, we focus only on the HOM heat ratio $\mathcal{R}_{Q}^{\mathrm{HOM}}$.

The plots for $\mathcal{R}_{Q}^{\mathrm{HOM}}$ in the integer and in the fractional case are compared in Fig. 4 for different values of $q$. In the integer case, a single smooth dip is present for all the values of $q$, confirming the phenomenology described for the finitetemperature case. For the strongly correlated case, at $q=1$ one observes a smooth profile, except for a small decrease close to $t_{D}=0.5$. Intriguingly, the oscillations observed in Fig. 3 for $q>1$ are widely enhanced in this regime, such that the HOM ratio displays zeros, whose number increases with $q$, in addition to the central one, and it can also reach negative values. We emphasize that the change of sign in $\mathcal{R}_{Q}^{\mathrm{HOM}}$ is entirely due to $\mathcal{S}_{Q}^{\mathrm{HOM}}$, since $\mathcal{S}_{Q}^{\mathrm{vac}}$ is negligible at very low temperature [see Eqs. (56) and (62)]. This is a remarkable difference with respect to the charge noise generated in the same configurations, since charge conservation fixes the sign of current-current correlations. On the contrary, it should be pointed out that the sign of heat noise is not constrained by any conservation law [85].

To complete our discussion, it is interesting to show that heat noise in a HOM setup, where both terminals 1 and 4 are driven, cannot be reproduced in a setup where a single-drive 

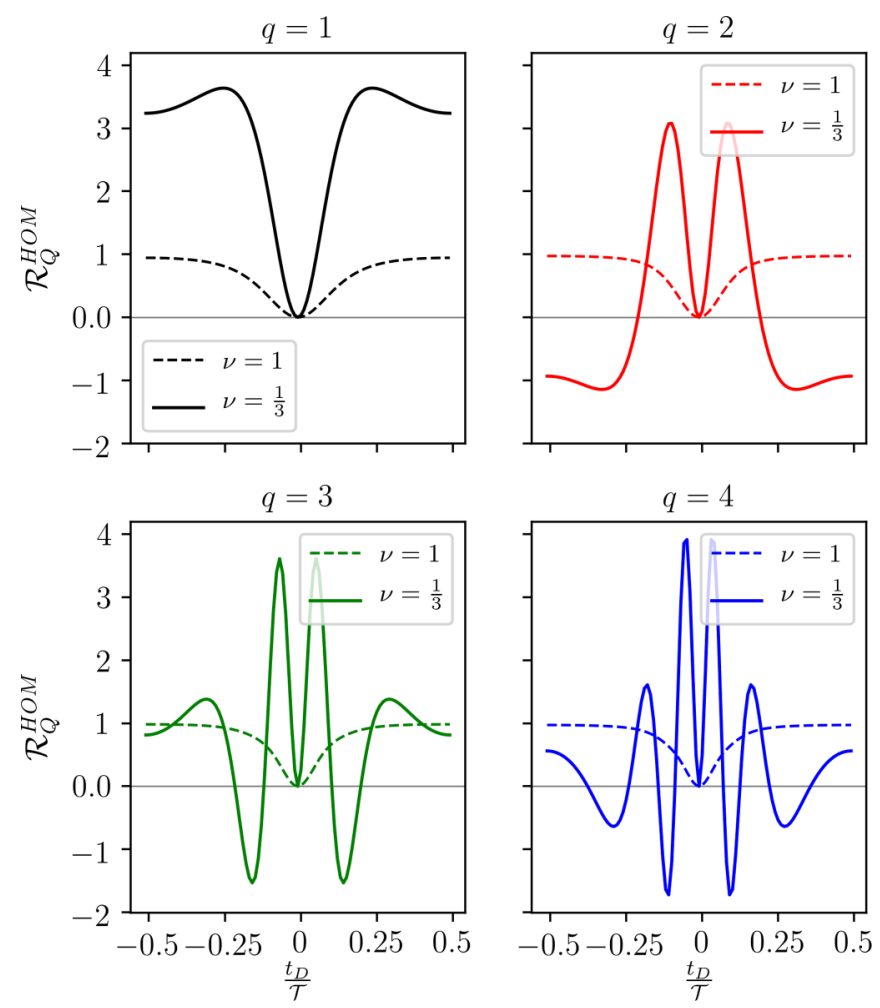

FIG. 4. HOM heat ratio $\mathcal{R}_{Q}^{\mathrm{HOM}}$ as a function of the time delay $t_{D}$ for $q=1,2,3$, and 4 . The integer case (dashed lines) and the fractional case for $v=\frac{1}{3}$ (solid lines) are compared. The other parameters are $W=0.1 \mathcal{T}, \theta=10^{-4} \omega$, and $\omega=0.01 \omega_{c}$.

$V_{-}(t)=V(t)-V\left(t+t_{D}\right)$ is applied exclusively to terminal 1. For this reason, we define an analogous ratio for single-drive heat noise as

$$
\mathcal{R}_{Q}^{\mathrm{sd}}=\frac{\mathcal{S}_{Q}^{\mathrm{sd}}-\mathcal{S}_{Q}^{\mathrm{vac}}}{2 \mathcal{S}_{Q}^{R}-2 \mathcal{S}_{Q}^{\mathrm{vac}}}
$$

We recall that by the notation $\mathcal{S}_{Q}^{\text {sd }}$ we label heat fluctuations generated in the specific setup where $V_{-}(t)$ is applied to a terminal and the other one is grounded.

In the upper panel of Fig. 5, $\mathcal{R}_{Q}^{\text {sd }}$ is presented for $q=1$ for two filling factors $v=1$ and $\frac{1}{3}$. Both temperature regimes, namely $\theta \sim \omega$ and $\theta \ll \omega$, are taken into account. Clearly, these plots are completely different from the ones shown in Fig. 2, further demonstrating that, in contrast to the charge case, heat noise generated in a HOM setup is not equivalent to the one generated in the presence of an effective single drive $V_{-}(t)$. In passing, let us also note that, as discussed for the HOM heat ratio, $\mathcal{R}_{O}^{\text {sd }}$ is not universal in temperature or filling factor, thus displaying another remarkable difference with respect to HOM charge noise.

Interestingly, we also note that single-drive ratio can switch sign as $t_{D}$ is tuned, independently of the filling factor. Since $\mathcal{S}_{Q}^{R}$ and $\mathcal{S}_{Q}^{\mathrm{vac}}$ are independent of $t_{D}$, the change of sign of $\mathcal{R}_{Q}^{\mathrm{sd}}$ is entirely due to $\mathcal{S}_{Q}^{\text {sd }}$ itself. This result implies that $\mathcal{S}_{Q}^{\text {sd }}$ can reach values below the equilibrium heat noise $\mathcal{S}_{Q}^{\text {vac }}$ [see Eq. (64)], but it does not tell anything about the change of sign of $\mathcal{S}_{Q}^{\text {sd }}$ itself. To acquire information about this point, in the lower
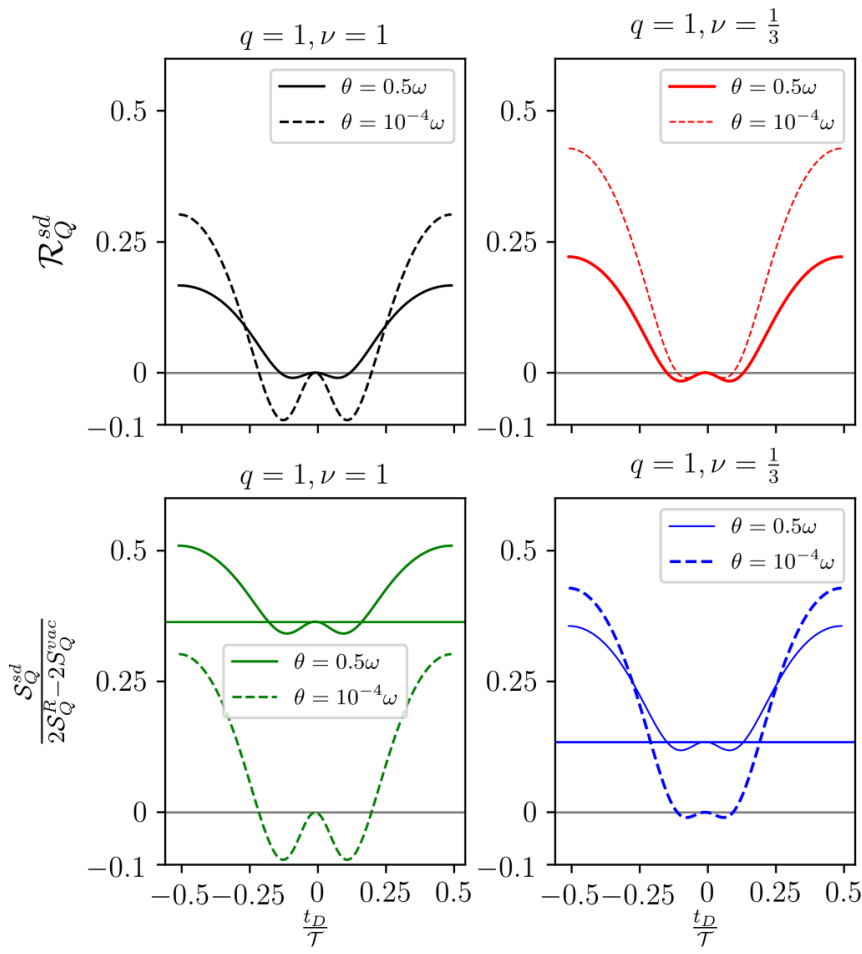

FIG. 5. Single-drive heat ratio $\mathcal{R}_{Q}^{\text {sd }}$ (upper panel) and single-drive heat noise $\mathcal{S}_{Q}^{\text {sd }}$ rescaled with respect to $2 \mathcal{S}_{Q}^{\text {sd }}-2 \mathcal{S}_{Q}^{\text {vac }}$, as a function of the time delay $t_{D}$ for $q=1$ and temperatures $\theta=0.25 \omega$ (solid lines) and $\theta=0.5 \omega$ (dashed lines). The integer case (left panel) and the fractional case for $v=\frac{1}{3}$ (right panel) are compared. The other parameters are $W=0.1 \mathcal{T}$ and $\omega=0.01 \omega_{c}$. In the lower panel, green and blue horizontal solid lines correspond to the values of $\frac{\mathcal{S}_{Q}^{\text {vac }}}{2 \mathcal{S}_{Q}^{\text {sd }}-2 \mathcal{S}_{Q}^{\text {vac }}}$ for $\theta=0.5 \omega$ at filling factors $v=1$ and $\frac{1}{3}$, respectively.

panel of Fig. 5 we show the plots for $\frac{\mathcal{S}_{Q}^{\text {sd }}}{2 \mathcal{S}_{0}^{\text {sd }}-2 \mathcal{S}_{Q}^{\text {vac }}}$ with the same parameters as the upper panel. One has to distinguish between the two temperature regimes. When $\theta \sim \omega$, the contribution of the equilibrium heat noise (solid green and blue horizontal lines) is still significant. As a consequence, single-drive heat noise is always positive, even though it goes below the equilibrium value for certain ranges of time delay. On the contrary, when $\theta \ll \omega, \mathcal{S}_{Q}^{\mathrm{vac}}$ is negligible with respect to other contributions, and the sign of $\mathcal{S}_{Q}^{\text {sd }}$ itself can be reversed by tuning the delay $t_{D}$.

\section{CONCLUSION}

In this work, we investigated charge and heat current fluctuations in an HOM interferometer in the fractional quantum Hall regime. Here, two identical leviton excitations impinge at a QPC with a given time delay. We started by evaluating zero-frequency cross-correlated charge and heat noises in the presence of two generic driving voltage $V_{1}$ and $V_{4}$. We demonstrated that heat noise in this double-drive configuration depends on both $V_{+}=V_{4}+V_{1}$ and $V_{-}=V_{1}-V_{4}$ and thus cannot be reproduced in a single-drive setup driven by the voltage $V_{-}$only. In particular, this implies that the singledrive configuration and the HOM interferometer implemented with voltage sources are two physically distinct experimental 
configurations. Moreover, we proved that the HOM heat ratio vanishes for a null time delay for both integer and fractional filling factors, despite the presence, in the latter case, of emergent fractionally charged quasiparticles. Finally, we investigated the form of HOM heat ratio for different regimes of temperatures. Interestingly, unexpected side dips emerged only in the fractional regime, which can be related to the crystallization mechanism recently predicted for levitons [96].

\section{ACKNOWLEDGMENTS}

This project has received funding from the European Union's Horizon 2020 research and innovation programme under the Marie Skłodowska-Curie Grant Agreement No. 754462. L.V. and M.S. acknowledge support from CNR SPIN through Seed project "Electron quantum optics with quantized energy packets." This work was granted access to the HPC resources of Aix-Marseille Université financed by the project Equip@Meso (Grant No. ANR-10-EQPX-29-01). It has been carried out in the framework of project "1shot reloaded" (Grant No. ANR-14-CE32-0017) and benefited from the support of the Labex ARCHIMEDE (Grant No. ANR-11-LABX0033), all funded by the "investissements d'avenir" French Government program managed by the French National Research Agency (ANR). The project leading to this publication has received funding from Excellence Initiative of AixMarseille University - A*MIDEX, a French "investissements d'avenir" programme.

\section{APPENDIX A: COUPLING TO THE GATE}

In this Appendix, we show that there is no gauge transformation able to link the equations of motion for the configurations with two driving voltages $V_{1}$ and $V_{4}$ and the configuration with an effective single drive $V_{-}=V_{1}-V_{4}$, presented in the main text.

In the double-drive setup, a voltage drive is applied both to right-moving and left-moving excitations. We consider a situation in which the vector potentials $\mathcal{A}_{R / L}(x, t)$ are absent. The Lagrangian density is

$$
\begin{aligned}
\mathcal{L}= & \frac{1}{4 \pi}\left\{-\partial_{x} \Phi_{R}(x, t)\left[\partial_{t} \Phi_{R}(x, t)+v \partial_{x} \Phi_{R}(x, t)\right]\right. \\
& \left.+\partial_{x} \Phi_{L}(x, t)\left[\partial_{t} \Phi_{L}(x, t)-v \partial_{x} \Phi_{L}(x, t)\right]\right\}
\end{aligned}
$$

$$
+\frac{e \sqrt{v}}{2 \pi}\left[\partial_{x} \Phi_{R}(x, t) \mathcal{V}_{1}(x, t)-\partial_{x} \Phi_{L}(x, t) \mathcal{V}_{4}(x, t)\right]
$$

The Euler-Lagrange equations

$$
\partial_{t} \frac{\delta \mathcal{L}}{\delta \partial_{t} \Phi_{\alpha}}+\partial_{x} \frac{\delta \mathcal{L}}{\delta \partial_{x} \Phi_{\alpha}}-\frac{\delta \mathcal{L}}{\delta \Phi_{\alpha}}=0
$$

with $\alpha=R, L$, give rise to the following equation of motions for the bosonic fields:

$$
\begin{aligned}
& \left(\partial_{t}+v \partial_{x}\right) \Phi_{R}(x, t)=e \sqrt{v} \mathcal{V}_{1}(x, t), \\
& \left(\partial_{t}-v \partial_{x}\right) \Phi_{L}(x, t)=e \sqrt{v} \mathcal{V}_{4}(x, t) .
\end{aligned}
$$

To model the system presented in Sec. II, the form for the voltage drives is

$$
\begin{aligned}
& \mathcal{V}_{1}(x, t)=f_{R}(x) V_{1}(t), \\
& \mathcal{V}_{4}(x, t)=f_{L}(x) V_{4}(t),
\end{aligned}
$$

where $f_{R / L}(x)$ are time-independent, while $V_{1 / 4}(t)$ are spaceindependent. In this case, the equations of motion for the double-drive setup are

$$
\begin{aligned}
& \left(\partial_{t}+v \partial_{x}\right) \Phi_{R}(x, t)=e \sqrt{v} f_{R}(x) V_{1}(t), \\
& \left(\partial_{t}-v \partial_{x}\right) \Phi_{L}(x, t)=e \sqrt{v} f_{L}(x) V_{4}(t) .
\end{aligned}
$$

We also consider a single-drive setup with an effective voltage drive $\mathcal{V}_{1}(x, t)=f_{R}(x)\left[V_{1}(t)-V_{4}(t)\right]$ on the right side, and the left side grounded $\left[\mathcal{V}_{4}(x, t)=0\right]$. We still consider that the magnetic potential is zero on both edges. It can be immediately seen that the equations of motion are now

$$
\begin{aligned}
\left(\partial_{t}+v \partial_{x}\right) \Phi_{R}(x, t) & =e \sqrt{v} f_{R}(x)\left[V_{1}(t)-V_{4}(t)\right], \\
\left(\partial_{t}-v \partial_{x}\right) \Phi_{L}(x, t) & =0 .
\end{aligned}
$$

\section{Applying gauge transformations to the HOM setup}

Here we show that a gauge transformation that operates in the following way on the voltage drives:

$$
\left\{\begin{array} { l } 
{ \mathcal { V } _ { 1 } ( x , t ) = f _ { R } ( x ) V _ { 1 } ( t ) , } \\
{ \mathcal { V } _ { 4 } ( x , t ) = f _ { L } ( x ) V _ { 4 } ( t ) , }
\end{array} \longrightarrow \left\{\begin{array}{l}
\mathcal{V}_{R}^{\prime}(x, t)=f_{R}(x)\left[V_{1}(t)-V_{4}(t)\right] \\
\mathcal{V}_{L}^{\prime}(x, t)=0
\end{array}\right.\right.
$$

does not transform Eqs. (A7) into Eqs. (A8), but leaves them unchanged.

We recall that a general gauge transformation that leaves invariant an electromagnetic field is given by

$$
\begin{array}{r}
\mathcal{V}_{R / L}^{\prime}(x, t)=\mathcal{V}_{1 / 4}(x, t)-\partial_{t} \chi_{R}(x, t), \\
\mathcal{A}_{R / L}^{\prime}(x, t)=\mathcal{A}_{R / L}(x, t)+\partial_{x} \chi_{R}(x, t),
\end{array}
$$

with $\chi_{R / L}(x, t)$ a scalar function.
In our particular case, voltage potentials are required to transform as

$$
\mathcal{V}_{R}^{\prime}(x, t)=f_{R}(x) V_{1}(x)-\partial_{t} \chi_{R}(x, t)=f_{R}(x)\left[V_{1}(t)-V_{4}(t)\right]
$$

$$
\mathcal{V}_{L}^{\prime}(x, t)=f_{L}(x) V_{4}(x)-\partial_{t} \chi_{L}(x, t)=0
$$

for the right-moving and left-moving sector, respectively. The transformation is evidently implemented by the 
choice

$$
\begin{aligned}
& \chi_{R}(x, t)=f_{R}(x) \int_{0}^{t} d \tau V_{4}(\tau), \\
& \chi_{L}(x, t)=f_{L}(x) \int_{0}^{t} d \tau V_{4}(\tau) .
\end{aligned}
$$

Since these equations involve spatial-dependent functions, we expect that nonzero magnetic potentials arise as a consequence of the gauge transformation. In the new gauge, we get nonzero magnetic potentials given by (in our initial gauge choice $\mathcal{A}_{R / L}=0$ )

$$
\begin{aligned}
& \mathcal{A}_{R}^{\prime}(x, t)=\partial_{x} f_{R}(x) \int_{0}^{t} d \tau V_{4}(\tau), \\
& \mathcal{A}_{L}^{\prime}(x, t)=\partial_{x} f_{L}(x) \int_{0}^{t} d \tau V_{4}(\tau),
\end{aligned}
$$

and the Lagrangian density now reads

$$
\begin{aligned}
\mathcal{L}^{\prime}= & \frac{1}{4 \pi}\left\{-\partial_{x} \Phi_{R}(x, t)\left[\partial_{t} \Phi_{R}(x, t)+v \partial_{x} \Phi_{R}(x, t)\right]\right. \\
& \left.+\partial_{x} \Phi_{L}(x, t)\left[\partial_{t} \Phi_{L}(x, t)-v \partial_{x} \Phi_{L}(x, t)\right]\right\} \\
& +\frac{e \sqrt{v}}{2 \pi}\left\{\partial_{x} \Phi_{R}(x, t) f_{R}(x)\left[V_{1}(t)-V_{4}(t)\right]\right. \\
& +\left[\partial_{t} \Phi_{R}(x, t) \partial_{x} f_{R}(x)-\partial_{t} \Phi_{L}(x, t) \partial_{x} f_{L}(x)\right] \\
& \left.\times \int_{t_{0}}^{t} d \tau V_{4}(\tau)\right\},
\end{aligned}
$$

where the last term accounts for the presence of $\mathcal{A}_{R}^{\prime}(x, t)$ and $\mathcal{A}_{L}^{\prime}(x, t)$. We now look for the equations of motion in this new configuration. From Euler-Lagrange equations, one gets

$$
\begin{aligned}
\left(\partial_{t}+\right. & \left.v \partial_{x}\right) \Phi_{R}(x, t) \\
= & e \sqrt{v} f_{R}(x)\left[V_{1}(t)-V_{4}(t)\right]+e \sqrt{v} f_{R}(x) V_{4}(t) \\
= & e \sqrt{v} f_{R}(x) V_{1}(t), \\
& \left(\partial_{t}-v \partial_{x}\right) \Phi_{L}(x, t)=e \sqrt{v} f_{L}(x) V_{4}(t) .
\end{aligned}
$$

Note that we have not recovered the equation of motions for the effective single drive setup, Eqs. (A8), as one may naively expect. On the contrary, we have found the equations of motion for the double-drive setup, Eqs. (A7).

\section{APPENDIX B: HEAT NOISE}

In this Appendix, we give more details about the calculation of heat noise presented in Sec. III. Before starting with the derivation of heat noise, we give some formulas that would be useful in the following parts.

\section{Useful formulas}

In the following, we derive some results that would be useful for the evaluation of heat current fluctuations. In particular, our goal is to evaluate the following average values (for simplicity, we drop all the low indices $R$ or $L$ ):

$$
\begin{aligned}
& C_{1}\left(t_{1}, t_{2}, t_{3}\right)=\left\langle\partial_{t_{1}} \phi\left(t_{1}\right) e^{i \sqrt{v} \phi\left(t_{2}\right)} e^{-i \sqrt{v} \phi\left(t_{3}\right)}\right\rangle, \\
& C_{2}\left(t_{1}, t_{2}, t_{3}\right)=\left\langle e^{i \sqrt{v} \phi\left(t_{1}\right)} e^{-i \sqrt{v} \phi\left(t_{2}\right)} \partial_{t_{3}} \phi\left(t_{3}\right)\right\rangle,
\end{aligned}
$$

$$
\begin{gathered}
D_{1}\left(t_{1}, t_{2}, t_{3}\right)=\left\langle\left[\partial_{t_{1}} \phi\left(t_{1}\right)\right]^{2} e^{i \sqrt{v} \phi\left(t_{2}\right)} e^{-i \sqrt{v} \phi\left(t_{3}\right)}\right\rangle, \\
D_{2}\left(t_{1}, t_{2}, t_{3}\right)=\left\langle e^{i \sqrt{v} \phi\left(t_{1}\right)} e^{-i \sqrt{v} \phi\left(t_{2}\right)}\left[\partial_{t_{3}} \phi\left(t_{3}\right)\right]^{2}\right\rangle,
\end{gathered}
$$

where the thermal average is performed over the initial equilibrium density matrix, in the absence of tunneling, and the driving voltage and bosonic fields evolve according to the edge Hamiltonian $H_{0}$. To evaluate $C_{1}$ and $C_{2}$, we start by considering the following general average value:

$$
E_{1}\left(\epsilon_{1}, \epsilon_{2}, \epsilon_{3} ; t_{1}, t_{2}, t_{3}\right)=\left\langle e^{-i \epsilon_{1} \phi\left(t_{1}\right)} e^{-i \epsilon_{2} \phi\left(t_{2}\right)} e^{-i \epsilon_{3} \phi\left(t_{3}\right)}\right\rangle,
$$

which is connected to $C_{1}$ and $C_{2}$ by this relation,

$$
\begin{aligned}
& C_{1}\left(t_{1}, t_{2}, t_{3}\right) \\
& \quad=i \partial_{t_{1}}\left\{\lim _{\epsilon_{1} \rightarrow 0} \partial_{\epsilon_{1}} E_{1}\left(\epsilon_{1}, \epsilon_{2}, \epsilon_{3} ; t_{1}, t_{2}, t_{3}\right)\right\} \begin{array}{c}
\epsilon_{2}=-\sqrt{v} \\
\epsilon_{3}=\sqrt{v}
\end{array} \\
& C_{2}\left(t_{1}, t_{2}, t_{3}\right) \\
& \quad=i \partial_{t_{3}}\left\{\lim _{\epsilon_{3} \rightarrow 0} \partial_{\epsilon_{3}} E_{1}\left(\epsilon_{1}, \epsilon_{2}, \epsilon_{3} ; t_{1}, t_{2}, t_{3}\right)\right\} \begin{array}{c}
\epsilon_{1}=-\sqrt{v} \\
\epsilon_{2}=\sqrt{v}
\end{array}
\end{aligned}
$$

By using [116]

$$
\left\langle e^{\chi\left(t_{1}\right)} e^{\chi\left(t_{2}\right)} e^{\chi\left(t_{3}\right)}\right\rangle=e^{\frac{1}{2} \sum_{i=1}^{3}\left\langle\chi\left(t_{i}\right)^{2}\right\rangle} e^{\sum_{i<j}\left\langle\chi\left(t_{i}\right) \chi\left(t_{j}\right)\right\rangle},
$$

we obtain from Eq. (B5)

$$
\begin{aligned}
& E_{1}\left(\epsilon_{1}, \epsilon_{2}, \epsilon_{3} ; x_{1}, x_{2}, x_{3}\right)=e^{-\frac{1}{2} \sum_{i=1}^{3}\left\langle\epsilon_{i}^{2} \phi^{2}\left(x_{i}\right)\right\rangle} \\
& \times e^{-\left\{\epsilon_{1} \epsilon_{2}\left\langle\phi\left(x_{1}\right) \phi\left(x_{2}\right)\right\rangle+\epsilon_{1} \epsilon_{3}\left\langle\phi\left(x_{1}\right) \phi\left(x_{3}\right)\right\rangle+\epsilon_{2} \epsilon_{3}\left\langle\phi\left(x_{2}\right) \phi\left(x_{3}\right)\right\rangle\right\}} .
\end{aligned}
$$

Finally, we use Eqs. (B6) and (B7) to find $C_{1}$ and $C_{2}$,

$$
\begin{gathered}
C_{1}\left(t_{1}, t_{2}, t_{3}\right)=-i \sqrt{\nu} \mathcal{K}\left(t_{1}, t_{2}, t_{3}\right) P_{\nu}\left(t_{2}-t_{3}\right), \\
C_{2}\left(t_{1}, t_{2}, t_{3}\right)=-i \sqrt{v} \mathcal{K}\left(-t_{3},-t_{1},-t_{2}\right) P_{\nu}\left(t_{1}-t_{2}\right),
\end{gathered}
$$

where we defined [see Eq. (19) in the main text]

$$
\begin{aligned}
P_{g}\left(t^{\prime}-t\right) & =\left\langle e^{i \sqrt{8} \phi_{R / L}\left(0, t^{\prime}\right)} e^{-i \sqrt{g} \phi_{R / L}(0, t)}\right\rangle \\
& =\left[\frac{\pi \theta \tau}{\sinh (\pi \theta \tau)\left(1+i \omega_{c} \tau\right)}\right]^{g}
\end{aligned}
$$

and

$$
\begin{aligned}
\mathcal{K}\left(t_{1}, t_{2}, t_{3}\right) & =\partial_{t_{1}}\left\{\left\langle\phi\left(t_{1}\right) \phi\left(t_{3}\right)\right\rangle-\left\langle\phi\left(t_{1}\right) \phi\left(t_{2}\right)\right\rangle\right\} \\
& =\int d \tau \mathcal{P}_{2}\left(t_{1}-\tau\right)\left[\Theta\left(\tau-t_{3}\right)-\Theta\left(\tau-t_{2}\right)\right] .
\end{aligned}
$$

One could also obtain the following similar relations:

$$
\begin{array}{r}
\left\langle\partial_{t_{1}} \phi\left(t_{1}\right) e^{-i \sqrt{\nu} \phi\left(t_{2}\right)} e^{i \sqrt{\nu} \phi\left(t_{3}\right)}\right\rangle=i \sqrt{\nu} \mathcal{K}\left(t_{1}, t_{2}, t_{3}\right) P_{v}\left(t_{2}-t_{3}\right), \\
\left\langle e^{-i \sqrt{\nu} \phi\left(t_{1}\right)} e^{i \sqrt{\nu} \phi\left(t_{2}\right) \partial_{t_{3}} \phi\left(t_{3}\right)}\right\rangle=i \sqrt{\nu} \mathcal{K}\left(-t_{3},-t_{1},-t_{2}\right) P_{\nu}\left(t_{1}-t_{2}\right) .
\end{array}
$$

Exploiting the following average:

$$
\left\langle\partial_{t_{1}} \phi\left(t_{1}\right) \partial_{t} \phi(t)\right\rangle=-\frac{\pi^{2} \theta^{2}}{v^{2} \sinh ^{2}\left[\pi \theta\left(t_{1}-t\right)\right]}
$$


the function $\mathcal{K}$ can be further evaluated by using

$$
\partial_{t_{1}}\left\langle\phi\left(t_{1}\right) \phi\left(t_{2}\right)\right\rangle=\int_{-\infty}^{t_{2}} d t\left\langle\partial_{t_{1}} \phi\left(t_{1}\right) \partial_{t} \phi(t)\right\rangle=\frac{\pi \theta}{v}\left\{\operatorname{coth}\left[\pi \theta\left(t_{1}-t_{2}\right)\right]-1\right\} .
$$

By using this result, one finds

$$
\mathcal{K}\left(t_{1}, t_{2}, t_{3}\right)=\frac{\pi \theta}{v}\left\{\operatorname{coth}\left[\pi \theta\left(t_{1}-t_{3}\right)\right]-\operatorname{coth}\left[\pi \theta\left(t_{1}-t_{2}\right)\right]\right\}=\frac{\pi \theta}{v} \frac{\sinh \left[\pi \theta\left(t_{2}-t_{3}\right)\right]}{\sinh \left[\pi \theta\left(t_{1}-t_{3}\right)\right] \sinh \left[\pi \theta\left(t_{1}-t_{2}\right)\right]} .
$$

To evaluate $D_{1}$ and $D_{2}$, we start by considering the following general average value:

$$
E_{2}\left(\epsilon_{1}, \epsilon_{2}, \epsilon_{3}, \epsilon_{4} ; t_{1}, t_{2}, t_{3}, t_{4}\right)=\left\langle e^{-i \epsilon_{1} \phi\left(t_{1}\right)} e^{-i \epsilon_{2} \phi\left(t_{2}\right)} e^{-i \epsilon_{3} \phi\left(t_{3}\right)} e^{-i \epsilon_{4} \phi\left(t_{4}\right)}\right\rangle,
$$

which is connected to $D_{1}$ and $D_{2}$ by these relations,

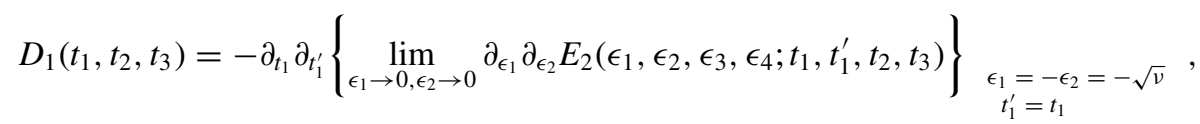

$$
\begin{aligned}
& D_{2}\left(t_{1}, t_{2}, t_{3}\right)=-\partial_{t_{3}} \partial_{t_{3}^{\prime}}\left\{\lim _{\epsilon_{1} \rightarrow 0, \epsilon_{2} \rightarrow 0} \partial_{\epsilon_{3}} \partial_{\epsilon_{4}} E_{2}\left(\epsilon_{1}, \epsilon_{2}, \epsilon_{3}, \epsilon_{4} ; t_{1}, t_{2}, t_{3}, t_{3}^{\prime}\right)\right\} \begin{array}{l}
t_{1}^{\prime}=t_{1} \\
\begin{array}{l}
\epsilon_{4}=-\epsilon_{3}=\sqrt{v} \\
t_{3}^{\prime}=t_{3}
\end{array}
\end{array} .
\end{aligned}
$$

By using Eq. (B8), we obtain from Eq. (B5)

$$
\begin{aligned}
& E_{2}\left(\epsilon_{1}, \epsilon_{2}, \epsilon_{3}, \epsilon_{4} ; t_{1}, t_{2}, t_{3}, t_{4}\right) \\
& \quad=e^{-\frac{1}{2} \sum_{i=1}^{4} \epsilon_{i}^{2}\left\langle\phi^{2}\left(t_{i}\right)\right\rangle} e^{-\left\{\epsilon_{1} \epsilon_{2}\left\langle\phi\left(t_{1}\right) \phi\left(t_{2}\right)\right\rangle+\epsilon_{1} \epsilon_{3}\left\langle\phi\left(t_{1}\right) \phi\left(t_{3}\right)\right\rangle+\epsilon_{1} \epsilon_{4}\left\langle\phi\left(t_{1}\right) \phi\left(t_{4}\right)\right\rangle+\epsilon_{2} \epsilon_{3}\left\langle\phi\left(t_{2}\right) \phi\left(t_{3}\right)\right\rangle+\epsilon_{2} \epsilon_{4}\left\langle\phi\left(t_{2}\right) \phi\left(t_{4}\right)\right\rangle+\epsilon_{3} \epsilon_{4}\left\langle\phi\left(t_{3}\right) \phi\left(t_{4}\right)\right\rangle\right\} .} .
\end{aligned}
$$

Finally, we use Eqs. (B20) and (B21) to find $D_{1}$ and $D_{2}$,

$$
\begin{gathered}
\left.\left[\partial_{t_{1}} \phi\left(t_{1}\right)\right]^{2} e^{i \sqrt{v} \phi\left(t_{2}\right)} e^{-i \sqrt{v} \phi\left(t_{3}\right)}\right\rangle=\left\{\left\langle\left[\partial_{t_{1}} \phi\left(t_{1}\right)\right]^{2}\right\rangle-v\left[\mathcal{K}\left(t_{1}, t_{2}, t_{3}\right)\right]^{2}\right\} P_{\nu}\left(t_{2}-t_{3}\right), \\
\left\langle e^{i \sqrt{v} \phi\left(t_{1}\right)} e^{-i \sqrt{v} \phi\left(t_{2}\right)}\left[\partial_{t_{3}} \phi\left(t_{3}\right)\right]^{2}\right\rangle=\left\{\left\langle\left[\partial_{t_{3}} \phi\left(t_{3}\right)\right]^{2}\right\rangle-v\left[\mathcal{K}\left(-t_{3},-t_{1},-t_{2}\right)\right]^{2}\right\} P_{\nu}\left(t_{1}-t_{2}\right) .
\end{gathered}
$$

By carrying on a similar calculation, one can find also the analogous quantities

$$
\begin{gathered}
\left.\left[\partial_{t_{1}} \phi\left(t_{1}\right)\right]^{2} e^{-i \sqrt{v} \phi\left(t_{2}\right)} e^{i \sqrt{v} \phi\left(t_{3}\right)}\right\rangle=\left\{\left\langle\left[\partial_{t_{1}} \phi\left(t_{1}\right)\right]^{2}\right\rangle-v\left[\mathcal{K}\left(t_{1}, t_{2}, t_{3}\right)\right]^{2}\right\} P_{\nu}\left(t_{2}-t_{3}\right), \\
\left\langle e^{-i \sqrt{v} \phi\left(t_{1}\right)} e^{i \sqrt{v} \phi\left(t_{2}\right)}\left[\partial_{t_{3}} \phi\left(t_{3}\right)\right]^{2}\right\rangle=\left\{\left\langle\left[\partial_{t_{3}} \phi\left(t_{3}\right)\right]^{2}\right\rangle-v\left[\mathcal{K}\left(-t_{3},-t_{1},-t_{2}\right)\right]^{2}\right\} P_{\nu}\left(t_{1}-t_{2}\right) .
\end{gathered}
$$

\section{Calculations of heat noise}

Our starting point is the perturbative expression of heat noise given in the main text [see Eq. (25)],

$$
\mathcal{S}_{Q}=\mathcal{S}_{Q}^{(02)}+\mathcal{S}_{Q}^{(20)}+\mathcal{S}_{Q}^{(11)}+O\left(|\Lambda|^{3}\right) .
$$

First, we derive the term $\mathcal{S}_{Q}^{(11)}$, which reads

$$
\mathcal{S}_{Q}^{(11)}=\int_{0}^{\mathcal{T}} \frac{d t}{\mathcal{T}} \int d t^{\prime}\left\{\left\langle\partial_{t^{\prime}} \Psi_{R}^{\dagger}\left(0, t^{\prime}\right) \Psi_{L}\left(0, t^{\prime}\right) \partial_{t} \Psi_{L}^{\dagger}(0, t) \Psi_{R}(0, t)\right\rangle+\left\langle\Psi_{L}^{\dagger}\left(0, t^{\prime}\right) \partial_{t^{\prime}} \Psi_{R}\left(0, t^{\prime}\right) \Psi_{R}^{\dagger}(0, t) \partial_{t} \Psi_{L}(0, t)\right\rangle\right\},
$$

since $\left\langle\mathcal{J}_{2 / 3}^{(1)}(t)\right\rangle=0$ [see Eq. (34) in the main text]. We recall that the time evolution of quasiparticle fields is

$$
\Psi_{R, L}(x, t)=\frac{\mathcal{F}_{R / L}}{\sqrt{2 \pi a}} e^{-i \sqrt{v} \phi_{R / L}(x, t)} e^{i v e \int_{t_{0}}^{t+\frac{x}{v}-\frac{d}{v}} d t^{\prime} V_{1 / 4}\left(t^{\prime}\right)} .
$$

We can further express the average in the above equation as

$$
\begin{aligned}
\mathcal{S}_{Q}^{(11)}= & 2|\lambda|^{2} \int_{0}^{\mathcal{T}} \frac{d t}{\mathcal{T}} \int d t^{\prime}\left\{\cos \left(v e \int_{t^{\prime}}^{t} d t^{\prime \prime} V_{1}\left(t^{\prime \prime}\right)-V_{4}\left(t^{\prime \prime}\right)\right) \partial_{t}^{\prime} \mathcal{P}_{v}\left(t^{\prime}-t\right) \partial_{t} \mathcal{P}_{v}\left(t^{\prime}-t\right)\right. \\
& +v e V_{1}\left(t^{\prime}\right) \sin \left(v e \int_{t^{\prime}}^{t} d t^{\prime \prime} V_{1}\left(t^{\prime \prime}\right)-V_{4}\left(t^{\prime \prime}\right)\right) \frac{1}{2} \partial_{t} \mathcal{P}_{2 v}\left(t^{\prime}-t\right)+v e V_{4}(t) \sin \left(v e \int_{t^{\prime}}^{t} d t^{\prime \prime} V_{1}\left(t^{\prime \prime}\right)-V_{4}\left(t^{\prime \prime}\right)\right) \frac{1}{2} \partial_{t^{\prime}} \mathcal{P}_{2 v}\left(t^{\prime}-t\right) \\
& \left.-v^{2} e^{2} V_{1}\left(t^{\prime}\right) V_{4}(t) \cos \left(v e \int_{t^{\prime}}^{t} d t^{\prime \prime} V_{1}\left(t^{\prime \prime}\right)-V_{4}\left(t^{\prime \prime}\right)\right) \mathcal{P}_{2 v}\left(t^{\prime}-t\right)\right\}
\end{aligned}
$$


where the function $P_{g}(t)$ is defined in Eq. (B12) and $\lambda \equiv \frac{\Lambda}{2 \pi a}$. The integration by parts of the second and third line of Eq. (B30) provides some useful eliminations, providing the final expression for this contribution,

$$
\begin{aligned}
\mathcal{S}_{Q}^{(11)}= & 2|\lambda|^{2} \int_{0}^{\mathcal{T}} \frac{d t}{\mathcal{T}} \int d t^{\prime}\left\{\cos \left(v e \int_{t^{\prime}}^{t} d t^{\prime \prime}\left[V_{1}\left(t^{\prime \prime}\right)-V_{4}\left(t^{\prime \prime}\right)\right]\right) \partial_{t}^{\prime} \mathcal{P}_{v}\left(t^{\prime}-t\right) \partial_{t} \mathcal{P}_{v}\left(t^{\prime}-t\right)\right. \\
& \left.-\frac{1}{2} v^{2} e^{2}\left[V_{1}\left(t^{\prime}\right) V_{1}(t)+V_{4}\left(t^{\prime}\right) V_{4}(t)\right] \cos \left(v e \int_{t^{\prime}}^{t} d t^{\prime \prime}\left[V_{1}\left(t^{\prime \prime}\right)-V_{4}\left(t^{\prime \prime}\right)\right]\right) \mathcal{P}_{2 v}\left(t^{\prime}-t\right)\right\} .
\end{aligned}
$$

We focus on the remaining contributions, starting from $\mathcal{S}_{Q}^{(02)}$ : the calculations for the other term would be analogous. By plugging Eqs. (26) and (28) in the definition of $\mathcal{S}_{Q}^{(02)}$, one finds

$$
\begin{aligned}
\mathcal{S}_{Q}^{(02)}= & -i \frac{|\lambda|^{2}}{4 \pi} \int d t \int_{0}^{\mathcal{T}} \frac{d t^{\prime}}{\mathcal{T}} \int d t^{\prime \prime} \theta\left(t^{\prime}-t^{\prime \prime}\right)\left\{\left\langle\left[\partial_{t} \phi_{R}(0, t)\right]^{2}\left[\Psi_{R}^{\dagger}\left(0, t^{\prime \prime}\right) \Psi_{L}\left(0, t^{\prime \prime}\right), \partial_{t^{\prime}} \Psi_{L}^{\dagger}\left(0, t^{\prime}\right) \Psi_{R}\left(0, t^{\prime}\right)\right]\right\rangle\right. \\
& -2 v e V_{1}(t)\left\langle\partial_{t} \phi_{R}(0, t)\left[\Psi_{R}^{\dagger}\left(0, t^{\prime \prime}\right) \Psi_{L}\left(0, t^{\prime \prime}\right), \partial_{t^{\prime}} \Psi_{L}^{\dagger}\left(0, t^{\prime}\right) \Psi_{R}\left(0, t^{\prime}\right)\right]\right\rangle \\
& \left.-\left\langle\left[\partial_{t} \phi_{R}(0, t)\right]^{2}\right\rangle\left\langle\left[\Psi_{R}^{\dagger}\left(0, t^{\prime \prime}\right) \Psi_{L}\left(0, t^{\prime \prime}\right), \partial_{t^{\prime}} \Psi_{L}^{\dagger}\left(0, t^{\prime}\right) \Psi_{R}\left(0, t^{\prime}\right)\right]\right\rangle\right\} .
\end{aligned}
$$

The averages involving the commutators can be performed by using the expression in Eq. (18) for the time evolution of quasiparticle fields and by resorting to the formulas in Eqs (B10), (B14), (B23), and (B24) derived in the Appendix B 1. Indeed, one finds

$$
\begin{aligned}
\mathcal{S}_{(Q)}^{(02)}= & -i \frac{|\lambda|^{2}}{4 \pi} \int d t \int_{0}^{\mathcal{T}} \frac{d t^{\prime}}{\mathcal{T}} \int d t^{\prime \prime} \Theta\left(t^{\prime}-t^{\prime \prime}\right)\left\{-\left[v \partial_{t^{\prime}} \mathcal{K}^{2}\left(t, t^{\prime}, t^{\prime \prime}\right) \cos \left(v e \int_{t^{\prime \prime}}^{t^{\prime}} d \tau V_{-}(\tau)\right)\left[\mathcal{P}_{2 v}\left(t^{\prime \prime}-t^{\prime}\right)-\mathcal{P}_{2 v}\left(t^{\prime}-t^{\prime \prime}\right)\right]\right.\right. \\
& \left.+v e V_{4}\left(t^{\prime}\right) \mathcal{K}^{2}\left(t, t^{\prime}, t^{\prime \prime}\right)\left[\mathcal{P}_{2 v}\left(t^{\prime \prime}-t^{\prime}\right)-\mathcal{P}_{2 v}\left(t^{\prime}-t^{\prime \prime}\right)\right] \sin \left(v e \int_{t^{\prime \prime}}^{t^{\prime}} d \tau V_{-}(\tau)\right)\right] \\
& +\mathcal{K}\left(t, t^{\prime}, t^{\prime \prime}\right)\left[2 v e V_{1}(t) \sin \left(v e \int_{t^{\prime \prime}}^{t^{\prime}} d \tau V_{-}(\tau)\right) \partial_{t^{\prime}}\left[\mathcal{P}_{2 v}\left(t^{\prime \prime}-t^{\prime}\right)-\mathcal{P}_{2 v}\left(t^{\prime}-t^{\prime \prime}\right)\right]\right. \\
& \left.\left.-4 v^{2} e^{2} V_{1}(t) V_{4}\left(t^{\prime}\right) \cos \left(v e \int_{t^{\prime \prime}}^{t^{\prime}} d \tau V_{-}(\tau)\right)\left[\mathcal{P}_{2 v}\left(t^{\prime \prime}-t^{\prime}\right)-\mathcal{P}_{2 v}\left(t^{\prime}-t^{\prime \prime}\right)\right]\right]\right\} .
\end{aligned}
$$

A similar calculation leads to the expression for the last contribution, given by

$$
\begin{aligned}
\mathcal{S}_{(Q)}^{(20)}= & -i \frac{|\lambda|^{2}}{4 \pi} \int d t \int_{0}^{\mathcal{T}} \frac{d t^{\prime}}{\mathcal{T}} \int d t^{\prime \prime} \Theta\left(t^{\prime}-t^{\prime \prime}\right)\left\{\left[v \partial_{t^{\prime}} \mathcal{K}^{2}\left(t, t^{\prime}, t^{\prime \prime}\right) \cos \left(v e \int_{t^{\prime \prime}}^{t^{\prime}} d \tau V_{-}(\tau)\right)\left[\mathcal{P}_{2 v}\left(t^{\prime \prime}-t^{\prime}\right)-\mathcal{P}_{2 v}\left(t^{\prime}-t^{\prime \prime}\right)\right]\right.\right. \\
& \left.+v e V_{4}\left(t^{\prime}\right) \mathcal{K}^{2}\left(t, t^{\prime}, t^{\prime \prime}\right)\left[\mathcal{P}_{2 v}\left(t^{\prime \prime}-t^{\prime}\right)-\mathcal{P}_{2 v}\left(t^{\prime}-t^{\prime \prime}\right)\right] \sin \left(v e \int_{t^{\prime \prime}}^{t^{\prime}} d \tau V_{-}(\tau)\right)\right] \\
& +\mathcal{K}\left(t, t^{\prime \prime}, t^{\prime}\right)\left[2 v e V_{4}(t) \sin \left(v e \int_{t^{\prime \prime}}^{t^{\prime}} d \tau V_{-}(\tau)\right) \partial_{t^{\prime}}\left[\mathcal{P}_{2 v}\left(t^{\prime \prime}-t^{\prime}\right)-\mathcal{P}_{2 v}\left(t^{\prime}-t^{\prime \prime}\right)\right]\right. \\
& \left.\left.+4 v^{2} e^{2} V_{1}(t) V_{4}\left(t^{\prime \prime}\right) \cos \left(v e \int_{t^{\prime \prime}}^{t^{\prime}} d \tau V_{-}(\tau)\right)\left[\mathcal{P}_{2 v}\left(t^{\prime \prime}-t^{\prime}\right)-\mathcal{P}_{2 v}\left(t^{\prime}-t^{\prime \prime}\right)\right]\right]\right\} .
\end{aligned}
$$

By summing up the two contributions, one can see that the first lines cancel out and the remaining two lines add up in a way that allows us to get rid of the function $\Theta\left(t^{\prime}-t^{\prime \prime}\right)$, thus obtaining

$$
\begin{aligned}
\mathcal{S}_{(Q)}^{(02)}+\mathcal{S}_{(Q)}^{(20)}= & -i \frac{|\lambda|^{2}}{4 \pi} \int d t \int_{0}^{\mathcal{T}} \frac{d t^{\prime}}{\mathcal{T}} \int d t^{\prime \prime}\left\{\mathcal { K } ( t , t ^ { \prime \prime } , t ^ { \prime } ) \left[2 v e V_{-}(t) \sin \left(v e \int_{t^{\prime \prime}}^{t^{\prime}} d \tau V_{-}(\tau)\right) \partial_{t^{\prime}}\left[\mathcal{P}_{2 v}\left(t^{\prime \prime}-t^{\prime}\right)-\mathcal{P}_{2 v}\left(t^{\prime}-t^{\prime \prime}\right)\right]\right.\right. \\
& \left.\left.-4 v^{2} e^{2} V_{1}(t) V_{4}\left(t^{\prime \prime}\right) \cos \left(v e \int_{t^{\prime \prime}}^{t^{\prime}} d \tau V_{-}(\tau)\right)\left[\mathcal{P}_{2 v}\left(t^{\prime \prime}-t^{\prime}\right)-\mathcal{P}_{2 v}\left(t^{\prime}-t^{\prime \prime}\right)\right]\right]\right\} .
\end{aligned}
$$

Now, summing all the contributions according to Eq. (38), it is possible to obtain the result presented in the main text, which reads

$$
\mathcal{S}_{Q}\left(V_{1}, V_{4}\right)=\mathcal{S}_{Q}\left(V_{-}, 0\right)+\Delta \mathcal{S}_{Q}\left(V_{1}, V_{4}\right),
$$


with

$$
\begin{gathered}
\mathcal{S}_{Q}\left(V_{-}, 0\right)=|\lambda|^{2} \int_{0}^{\mathcal{T}} \frac{d t}{\mathcal{T}} \int d t^{\prime}\left\{\cos \left(v e \int_{t}^{t^{\prime}} d \tau V_{-}(\tau)\right) \operatorname{Re}\left[\mathcal{P}_{v}\left(t^{\prime}-t\right) \partial_{t}^{2} \mathcal{P}_{v}\left(t^{\prime}-t\right)\right]\right. \\
\left.+\frac{v e v}{\pi} \int d t^{\prime \prime} V_{-}\left(t^{\prime}\right) \mathcal{K}\left(t^{\prime}, t, t^{\prime \prime}\right) \sin \left(v e \int_{t}^{t^{\prime}} d \tau V_{-}(\tau)\right) \operatorname{Im}\left[\partial_{t^{\prime \prime}} \mathcal{P}_{2 v}\left(t^{\prime \prime}-t\right)\right]\right\} \\
\Delta \mathcal{S}_{Q}\left(V_{1}, V_{4}\right)=v^{2} e^{2}|\lambda|^{2} \int_{0}^{\mathcal{T}} \frac{d t}{\mathcal{T}} \int d t^{\prime} \cos \left(v e \int_{t}^{t^{\prime}} d \tau V_{-}(\tau)\right)\left(\alpha_{R L}\left(t, t^{\prime}\right) \operatorname{Re}\left[\mathcal{P}_{2 v}\left(t^{\prime}-t\right)\right]+\beta_{R L}\left(t, t^{\prime}\right) \operatorname{Im}\left[\mathcal{P}_{2 v}\left(t^{\prime}-t\right)\right]\right),
\end{gathered}
$$

where we defined the following functions:

$$
\begin{gathered}
\alpha_{R L}\left(t, t^{\prime}\right)=\left[V_{1}(t) V_{4}\left(t^{\prime}\right)-V_{4}(t) V_{1}\left(t^{\prime}\right)\right], \\
\beta_{R L}=\frac{v}{\pi} \int d t^{\prime \prime} \mathcal{K}\left(t^{\prime \prime}, t, t^{\prime}\right) V_{1}\left(t^{\prime \prime}\right)\left[V_{4}\left(t^{\prime}\right)-V_{4}(t)\right] .
\end{gathered}
$$

\section{APPENDIX C: FOURIER COEFFICIENTS}

This Appendix is devoted to the Fourier analysis of the Lorentzian periodic signal $V_{\mathrm{Lor}}(t)$ and of the phase $e^{-i v e \int_{t_{0}}^{t} d t^{\prime} V_{\text {Lor }}\left(t^{\prime}\right)}$, where

$$
V_{\text {Lor }}(t)=\frac{V_{0}}{\pi} \sum_{k=-\infty}^{+\infty} \frac{W}{W^{2}+(t-k \mathcal{T})^{2}},
$$

where $\mathcal{T}$ is the periodic, $V_{0}$ the amplitude, and $W$ the halfwidth at half-maximum.

The coefficients for the Fourier series of the expression $v e V_{\text {Lor }}(t)=\sum_{k} c_{k} e^{i k \omega t}$ are

$$
c_{k}=q \omega e^{-2 \pi \frac{W}{T}|k|},
$$

with $q=\frac{\nu e}{2 \pi} \int_{0}^{\mathcal{T}} d t V_{\mathrm{Lor}}(t)=\frac{v e V_{0}}{\omega}$.

We also note that, for the time-delayed voltage $V_{\mathrm{Lor}}\left(t+t_{D}\right)$, the coefficients become $c_{k}^{\prime}=c_{k} e^{-i k \omega t_{D}}$.

The Fourier series $e^{-i v e \int_{0}^{t} d t^{\prime}\left[V_{\text {Lor }}\left(t^{\prime}\right)-V_{0}\right]}=\sum_{l} p_{l} e^{-i l \omega t}$ allows us to deal with the time-dependent problem as a superposition of time-independent configurations, with energy shifted by an integer amount of energy quanta $\omega$. For the Lorentzian case, it is convenient to switch to a complex representation in terms of the variable $z=e^{i \omega t}$. After some algebra and introducing $\gamma=e^{-2 \pi \eta}$, one finds [3,4]

$$
p_{l}=\frac{1}{2 \pi i} \oint_{|z|=1} d z z^{l+q-1}\left(\frac{1-z \gamma}{z-\gamma}\right)^{q} .
$$

From Eq. (C3) one can make use of complex binomial series and Cauchy's integral theorem $[117,118]$ to finally get

$$
p_{l}=q \gamma^{l} \sum_{s=0}^{\infty}(-1)^{s} \frac{\Gamma(l+s+q)}{\Gamma(1+q-s)} \frac{\gamma^{2 s}}{s !(s+l) !} .
$$

Finally, the Fourier coefficients $\tilde{p_{l}}$ for the voltage phase $e^{-i v e \int_{0}^{t} d \tau\left[V_{\mathrm{Lor}}(\tau)-V_{\mathrm{Lor}}\left(\tau+t_{D}\right)\right]}$ in the HOM configuration are given by

$$
\tilde{p_{l}}=\int_{0}^{\mathcal{T}} \frac{d t}{\mathcal{T}} e^{i l \omega t} e^{-i v e \int_{0}^{t} d \tau\left[V_{\mathrm{Lor}}(\tau)-V_{\mathrm{Lor}}\left(\tau+t_{D}\right)\right]},
$$

which can be calculated in terms of the coefficient $p_{l}$ of an effective single drive as

$$
\tilde{p_{l}}=\sum_{m} p_{m}^{*} p_{m+l} e^{-i m \omega t_{D}}
$$

[1] E. Bocquillon, V. Freulon, F. D. Parmentier, J.-M. Berroir, B. Plaçais, C. Wahl, J. Rech, T. Jonckheere, T. Martin, C. Grenier, D. Ferraro, P. Degiovanni, and G. Fève, Electron quantum optics in ballistic chiral conductors, Ann. Phys. (Berlin) 526, 1 (2014).

[2] C. Grenier, R. Hervé, G. Fève, and P. Degiovanni, Electron quantum optics in quantum Hall edge channels, Mod. Phys. Lett. B 25, 1053 (2011).

[3] J. Dubois, T. Jullien, C. Grenier, P. Degiovanni, P. Roulleau, and D. C. Glattli, Integer and fractional charge Lorentzian voltage pulses analyzed in the framework of photon-assisted shot noise, Phys. Rev. B 88, 085301 (2013).

[4] C. Grenier, J. Dubois, T. Jullien, P. Roulleau, D. C. Glattli, and P. Degiovanni, Fractionalization of minimal excitations in integer quantum Hall edge channels, Phys. Rev. B 88, 085302 (2013).
[5] M. Misiorny, G. Fève, and J. Splettstoesser, Shaping charge excitations in chiral edge states with a time-dependent gate voltage, Phys. Rev. B 97, 075426 (2018).

[6] D. C. Glattli and P. Roulleau, Levitons for electron quantum optics, Phys. Status Solidi B 254, 1600650 (2017).

[7] C. Bäuerle, D. C. Glattli, T. Meunier, F. Portier, P. Roche, P. Roulleau, S. Takada, and X. Waintal, Coherent control of single electrons: a review of current progress, Rep. Prog. Phys. 81, 056503 (2018).

[8] M. Büttiker, Capacitance, admittance, and rectification properties of small conductors, J. Phys.: Condens. Matter 5, 9361 (1993).

[9] M. Büttiker, H. Thomas, and A. Prêtre, Mesoscopic capacitors, Phys. Lett. A 180, 364 (1993).

[10] M. Moskalets, Noise of a single-electron emitter, Phys. Rev. B 88, 035433 (2013). 
[11] G. Fève, A. Mahé, J.-M. Berroir, T. Kontos, B. Plaçais, D. C. Glattli, A. Cavanna, B. Etienne, and Y. Jin, An on-demand coherent single-electron source, Science 316, 1169 (2007).

[12] E. Bocquillon, F. D. Parmentier, C. Grenier, J.-M. Berroir, P. Degiovanni, D. C. Glattli, B. Plaçais, A. Cavanna, Y. Jin, and G. Fève, Electron Quantum Optics: Partitioning Electrons One by One, Phys. Rev. Lett. 108, 196803 (2012).

[13] F. D. Parmentier, E. Bocquillon, J.-M. Berroir, D. C. Glattli, B. Plaçais, G. Fève, M. Albert, C. Flindt, and M. Büttiker, Current noise spectrum of a single-particle emitter: Theory and experiment, Phys. Rev. B 85, 165438 (2012).

[14] D. Ferraro, J. Rech, T. Jonckheere, and T. Martin, Single quasiparticle and electron emitter in the fractional quantum Hall regime, Phys. Rev. B 91, 205409 (2015).

[15] D. C. Glattli and P. Roulleau, Hanbury-Brown Twiss noise correlation with time controlled quasi-particles in ballistic quantum conductors, Physica E 76, 216 (2016).

[16] D. Ferraro, F. Ronetti, L. Vannucci, M. Acciai, J. Rech, T. Jockheere, T. Martin, and M. Sassetti, Hong-Ou-Mandel characterization of multiply charged Levitons, Eur. Phys. J. Spec. Top. 227, 1345 (2018).

[17] M. Moskalets, Fractionally Charged Zero-Energy SingleParticle Excitations in a Driven Fermi Sea, Phys. Rev. Lett. 117, 046801 (2016).

[18] I. Safi, Time-dependent transport in arbitrary extended driven tunnel junctions, arXiv:1401.5950 [cond-mat.mes-hall].

[19] F. Dolcini, Interplay between Rashba interaction and electromagnetic field in the edge states of a two-dimensional topological insulator, Phys. Rev. B 95, 085434 (2017).

[20] L. S. Levitov, H. Lee, and G. B. Lesovik, Electron counting statistics and coherent states of electric current, J. Math. Phys. 37, 4845 (1996).

[21] D. A. Ivanov, H. W. Lee, and L. S. Levitov, Coherent states of alternating current, Phys. Rev. B 56, 6839 (1997).

[22] J. Keeling, I. Klich, and L. S. Levitov, Minimal Excitation States of Electrons in One-Dimensional Wires, Phys. Rev. Lett. 97, 116403 (2006).

[23] M. Moskalets, First-order correlation function of a stream of single-electron wave packets, Phys. Rev. B 91, 195431 (2015).

[24] D. C. Glattli and P. Roulleau, Pseudorandom binary injection of levitons for electron quantum optics, Phys. Rev. B 97, 125407 (2018).

[25] D. Dasenbrook and C. Flindt, Dynamical generation and detection of entanglement in neutral leviton pairs, Phys. Rev. B 92, 161412(R) (2015).

[26] D. Dasenbrook, J. Bowles, J. B. Brask, P. Hofer, C. Flindt, and N. Brunner, Single-electron entanglement and nonlocality, New J. Phys. 18, 043036 (2016).

[27] D. Dasenbrook and C. Flindt, Dynamical Scheme for Interferometric Measurements of Full-Counting Statistics, Phys. Rev. Lett. 117, 146801 (2016).

[28] D. Ferraro, F. Ronetti, J. Rech, T. Jonckheere, M. Sassetti, and T. Martin, Enhancing photon squeezing one leviton at a time, Phys. Rev. B 97, 155135 (2018).

[29] C. Grenier, R. Hervé, E. Bocquillon, F. D. Parmentier, B. Plaçais, J. M. Berroir, G. Fève, and P. Degiovanni, Singleelectron quantum tomography in quantum Hall edge channels, New J. Phys. 13, 093007 (2011).

[30] D. Ferraro, A. Feller, A. Ghibaudo, E. Thibierge, E. Bocquillon, G. Fève, C. Grenier, and P. Degiovanni, Wigner function approach to single electron coherence in quantum Hall edge channels, Phys. Rev. B 88, 205303 (2013).

[31] D. Ferraro, B. Roussel, C. Cabart, E. Thibierge, G. Fève, Ch. Grenier, and P. Degiovanni, Real-Time Decoherence of Landau and Levitov Quasiparticles in Quantum Hall Edge Channels, Phys. Rev. Lett. 113, 166403 (2014).

[32] T. Jullien, P. Roulleau, B. Roche, A. Cavanna, Y. Jin, and D. C. Glattli, Quantum tomography of an electron, Nature (London) 514, 603 (2014).

[33] S. Ol'khovskaya, J. Splettstoesser, M. Moskalets, and M. Büttiker, Shot Noise of a Mesoscopic Two-Particle Collider, Phys. Rev. Lett. 101, 166802 (2008).

[34] G. Rosselló, F. Battista, M. Moskalets, and J. Splettstoesser, Interference and multiparticle effects in a Mach-Zehnder interferometer with single-particle sources, Phys. Rev. B 91, 115438 (2015).

[35] R. Hanbury Brown and R. Q. Twiss, Correlation between photons in two coherent beams of light, Nature (London) 177, 27 (1956).

[36] T. Martin, Noise in mesoscopic physics, in Nanophysics: Coherence and Transport. Les Houches Session LXXXI, edited by H. Bouchiat, Y. Gefen, S. Guéron, G. Montambaux, and J. Dalibard (Elsevier, Amsterdam, 2005), pp. 283-359.

[37] M. Moskalets, Single-particle shot noise at nonzero temperature, Phys. Rev. B 96, 165423 (2017).

[38] J. Dubois, T. Jullien, F. Portier, P. Roche, A. Cavanna, Y. Jin, W. Wegscheider, P. Roulleau, and D. C. Glattli, Minimalexcitation states for electron quantum optics using levitons, Nature (London) 502, 659 (2013).

[39] J. Rech, D. Ferraro, T. Jonckheere, L. Vannucci, M. Sassetti, and T. Martin, Minimal Excitations in the Fractional Quantum Hall Regime, Phys. Rev. Lett. 118, 076801 (2017).

[40] C. K. Hong, Z. Y. Ou, and L. Mandel, Measurement of Subpicosecond Time Intervals Between Two Photons by Interference, Phys. Rev. Lett. 59, 2044 (1987).

[41] E. Bocquillon, V. Freulon, J.-M Berroir, P. Degiovanni, B. Plaçais, A. Cavanna, Y. Jin, and G. Fève, Coherence and indistinguishability of single electrons emitted by independent sources, Science 339, 1054 (2013).

[42] T. Jonckheere, J. Rech, C. Wahl, and T. Martin, Electron and hole Hong-Ou-Mandel interferometry, Phys. Rev. B 86, 125425 (2012).

[43] D. Ferraro, C. Wahl, J. Rech, T. Jonckheere, and T. Martin, Electronic Hong-Ou-Mandel interferometry in twodimensional topological insulators, Phys. Rev. B 89, 075407 (2014).

[44] C. Wahl, J. Rech, T. Jonckheere, and T. Martin, Interactions and Charge Fractionalization in an Electronic Hong-OuMandel Interferometer, Phys. Rev. Lett. 112, 046802 (2014).

[45] V. Freulon, A. Marguerite, J.-M. Berroir, B. Plaçais, A. Cavanna, Y. Jin, and G. Fève, Hong-Ou-Mandel experiment for temporal investigation of single-electron fractionalization, Nat. Commun. 6, 6854 (2015).

[46] A. Marguerite, C. Cabart, C. Wahl, B. Roussel, V. Freulon, D. Ferraro, Ch. Grenier, J.-M. Berroir, B. Plaçais, T. Jonckheere, J. Rech, T. Martin, P. Degiovanni, A. Cavanna, Y. Jin, and G. Fève, Decoherence and relaxation of a single electron in a onedimensional conductor, Phys. Rev. B 94, 115311 (2016).

[47] C. Cabart, B. Roussel, G. Fève, and P. Degiovanni, Taming electronic decoherence in 1D chiral ballistic 
quantum conductors, Phys. Rev. B 98, 155302 (2018).

[48] L. Bellentani, P. Bordone, X. Oriols, and A. Bertoni, Coulomb and exchange interaction effects on the exact two-electron dynamics in the Hong-Ou-Mandel interferometer based on Hall edge states, arXiv:1903.02581 [cond-mat.mes-hall].

[49] F. Giazotto, T. T. Heikkilä, A. Luukanen, A. M. Savin, and J. P. Pekola, Opportunities for mesoscopics in thermometry and refrigeration: Physics and applications, Rev. Mod. Phys. 78, 217 (2006).

[50] R. Landauer, The noise is the signal, Nature 392, 658 (1998).

[51] M. Esposito, U. Harbola, and S. Mukamel, Nonequilibrium fluctuations, fluctuation theorems, and counting statistics in quantum systems, Rev. Mod. Phys. 81, 1665 (2009).

[52] M. Campisi, P. Hänggi, and P. Talkner, Colloquium: Quantum fluctuation relations: Foundations and applications, Rev. Mod. Phys. 83, 771 (2011).

[53] G. Benenti, G. Casati, K. Saito, and R. S. Whitney, Fundamental aspects of steady-state conversion of heat to work at the nanoscale, Phys. Rep. 694, 1 (2017).

[54] N. Li, J. Ren, L. Wang, G. Zhang, P. Hänggi, and B. Li, Colloquium: Phononics: Manipulating heat flow with electronic analogs and beyond, Rev. Mod. Phys. 84, 1045 (2012).

[55] F. Giazotto and M. J. Martínez-Pérez, The Josephson heat interferometer, Nature (London) 492, 401 (2012).

[56] M. J. Martínez-Pérez, A. Fornieri, and F. Giazotto, Rectification of electronic heat current by a hybrid thermal diode, Nat. Nanotechnol. 10, 303 (2015).

[57] A. Fornieri, G. Timossi, P. Virtanen, P. Solinas, and F. Giazotto, $0-\pi$ phase-controllable thermal Josephson junction, Nat. Nanotechnol. 12, 425 (2017).

[58] G. Granger, J. P. Eisenstein, and J. L. Reno, Observation of Chiral Heat Transport in the Quantum Hall Regime, Phys. Rev. Lett. 102, 086803 (2009).

[59] C. Altimiras, H. le Sueur, U. Gennser, A. Cavanna, D. Mailly, and F. Pierre, Tuning Energy Relaxation Along Quantum Hall Channels, Phys. Rev. Lett. 105, 226804 (2010).

[60] C. Altimiras, H. le Sueur, U. Gennser, A. Anthore, A. Cavanna, D. Mailly, and F. Pierre, Chargeless Heat Transport in the Fractional Quantum Hall Regime, Phys. Rev. Lett. 109, 026803 (2012).

[61] S. Jezouin, F. D. Parmentier, A. Anthore, U. Gennser, A. Cavanna, Y. Jin, and F. Pierre, Quantum limit of heat flow across a single electronic channel, Science 342, 601 (2013).

[62] C. L. Kane and M. P. A. Fisher, Quantized thermal transport in the fractional quantum Hall effect, Phys. Rev. B 55, 15832 (1997).

[63] M. Banerjee, M. Heiblum, A. Rosenblatt, Y. Oreg, D. E. Feldman, A. Stern, and V. Umansky, Observed quantization of anyonic heat flow, Nature (London) 545, 75 (2017).

[64] M. Banerjee, M. Heiblum, V. Umansky, D. E. Feldman, Y. Oreg, and A. Stern, Observation of half-integer thermal Hall conductance, Nature (London) 559, 205 (2018).

[65] S. H. Simon, Interpretation of thermal conductance of the $v=5 / 2$ edge, Phys. Rev. B 97, 121406(R) (2018).

[66] C. Wang, A. Vishwanath, and B. I. Halperin, Topological order from disorder and the quantized Hall thermal metal: Possible applications to the $v=5 / 2$ state, Phys. Rev. B 98, 045112 (2018).
[67] D. F. Mross, Y. Oreg, A. Stern, G. Margalit, and M. Heiblum, Theory of Disorder-Induced Half-Integer Thermal Hall Conductance, Phys. Rev. Lett. 121, 026801 (2018).

[68] B. Lian and J. Wang, Theory of the disordered $v=\frac{5}{2}$ quantum thermal Hall state: Emergent symmetry and phase diagram, Phys. Rev. B 97, 165124 (2018).

[69] R. Sánchez, B. Sothmann, and A. N. Jordan, Chiral Thermoelectrics with Quantum Hall Edge States, Phys. Rev. Lett. 114, 146801 (2015).

[70] R. Sánchez, B. Sothmann, and A. N. Jordan, Heat diode and engine based on quantum Hall edge states, New J. Phys. 17, 075006 (2015).

[71] P. Samuelsson, S. Kheradsoud, and B. Sothmann, Optimal Quantum Interference Thermoelectric Heat Engine with Edge States, Phys. Rev. Lett. 118, 256801 (2017).

[72] H. Thierschmann, R. Sánchez, B. Sothmann, F. Arnold, C. Heyn, W. Hansen, H. Buhmann, and L. W. Molenkamp, Threeterminal energy harvester with coupled quantum dots, Nat. Nanotechnol. 10, 854 (2015).

[73] S. Juergens, F. Haupt, M. Moskalets, and J. Splettstoesser, Thermoelectric performance of a driven double quantum dot, Phys. Rev. B 87, 245423 (2013).

[74] P. A. Erdman, F. Mazza, R. Bosisio, G. Benenti, R. Fazio, and F. Taddei, Thermoelectric properties of an interacting quantum dot based heat engine, Phys. Rev. B 95, 245432 (2017).

[75] F. Mazza, R. Bosisio, G. Benenti, V. Giovannetti, R. Fazio, and F. Taddei, Thermoelectric efficiency of threeterminal quantum thermal machines, New J. Phys. 16, 085001 (2014).

[76] F. Ronetti, M. Carrega, D. Ferraro, J. Rech, T. Jonckheere, T. Martin, and M. Sassetti, Polarized heat current generated by quantum pumping in two-dimensional topological insulators, Phys. Rev. B 95, 115412 (2017).

[77] M. F. Ludovico, J. S. Lim, M. Moskalets, L. Arrachea, and D. Sánchez, Dynamical energy transfer in ac-driven quantum systems, Phys. Rev. B 89, 161306(R) (2014).

[78] M. F. Ludovico, M. Moskalets, D. Sánchez, and L. Arrachea, Dynamics of energy transport and entropy production in acdriven quantum electron systems, Phys. Rev. B 94, 035436 (2016).

[79] M. Ludovico, L. Arrachea, M. Moskalets, and D. Sánchez, Probing the energy reactance with adiabatically driven quantum dots, Phys. Rev. B 97, 041416(R) (2018).

[80] F. Mazza, S. Valentini, R. Bosisio, G. Benenti, V. Giovannetti, R. Fazio, and F. Taddei, Separation of heat and charge currents for boosted thermoelectric conversion, Phys. Rev. B 91, 245435 (2015).

[81] M. Carrega, P. Solinas, A. Braggio, M. Sassetti, and U. Weiss, Functional integral approach to time-dependent heat exchange in open quantum systems: general method and applications, New J. Phys. 17, 045030 (2015).

[82] M. Carrega, P. Solinas, M. Sassetti, and U. Weiss, Energy Exchange in Driven Open Quantum Systems at Strong Coupling, Phys. Rev. Lett. 116, 240403 (2016).

[83] M. Campisi, P. Talkner, and P. Hänggi, Fluctuation Theorem for Arbitrary Open Quantum Systems, Phys. Rev. Lett. 102, 210401 (2009).

[84] M. Campisi, J. Pekola, and R. Fazio, Nonequilibrium fluctuations in quantum heat engines: theory, example, and possible solid state experiments, New J. Phys. 17, 035012 (2015). 
[85] M. Moskalets, Floquet Scattering Matrix Theory of Heat Fluctuations in Dynamical Quantum Conductors, Phys. Rev. Lett. 112, 206801 (2014).

[86] D. V. Averin and J. P. Pekola, Violation of the FluctuationDissipation Theorem in Time-Dependent Mesoscopic Heat Transport, Phys. Rev. Lett. 104, 220601 (2010).

[87] M. Moskalets and G. Haack, Heat and charge transport measurements to access single-electron quantum characteristics, Phys. Status Solidi B 254, 1600616 (2017).

[88] A. Crépieux and F. Michelini, Mixed, charge and heat noises in thermoelectric nanosystems, J. Phys.: Condens. Matter 27, 015302 (2014).

[89] A. Crépieux and F. Michelini, Heat-charge mixed noise and thermoelectric efficiency fluctuations, J. Stat. Mech. (2016) 054015 .

[90] F. Battista, F. Haupt, and J. Splettstoesser, Correlations between charge and energy current in ac-driven coherent conductors, J. Phys.: Conf. Ser. 568, 052008 (2014).

[91] F. Battista, M. Moskalets, M. Albert, and P. Samuelsson, Quantum Heat Fluctuations of Single-Particle Sources, Phys. Rev. Lett. 110, 126602 (2013).

[92] F. Battista, F. Haupt, and J. Splettstoesser, Energy and power fluctuations in ac-driven coherent conductors, Phys. Rev. B 90, 085418 (2014).

[93] L. Vannucci, F. Ronetti, J. Rech, D. Ferraro, T. Jonckheere, T. Martin, and M. Sassetti, Minimal excitation states for heat transport in driven quantum Hall systems, Phys. Rev. B 95, 245415 (2017).

[94] N. Dashti, M. Misiorny, P. Samuelsson, and J. Splettstoesser, Probing charge- and heat-current noise by frequencydependent fluctuations in temperature and potential, Phys. Rev. Appl. 10, 024007 (2018).

[95] R. B. Laughlin, Anomalous Quantum Hall Effect: An Incompressible Quantum Fluid with Fractionally Charged Excitations, Phys. Rev. Lett. 50, 1395 (1983).

[96] F. Ronetti, L. Vannucci, D. Ferraro, T. Jonckheere, J. Rech, T. Martin, and M. Sassetti, Crystallization of levitons in the fractional quantum Hall regime, Phys. Rev. B 98, 075401 (2018).

[97] X. G. Wen, Chiral Luttinger liquid and the edge excitations in the fractional quantum Hall states, Phys. Rev. B 41, 12838 (1990).

[98] X.-G. Wen, Topological orders and edge excitations in fractional quantum Hall states, Adv. Phys. 44, 405 (1995).

[99] F. Dolcini, R. C. Iotti, A. Montorsi, and F. Rossi, Photoexcitation of electron wave packets in quantum spin Hall edge states: Effects of chiral anomaly from a localized electric pulse, Phys. Rev. B 94, 165412 (2016).

[100] F. Dolcini and F. Rossi, Photoexcitation in two-dimensional topological insulators, Eur. Phys. J. Spec. Top. 227, 1323 (2018).
[101] C. Fleckenstein, N. Traverso Ziani, and B. Trauzettel, Chiral anomaly in real space from stable fractional charges at the edge of a quantum spin Hall insulator, Phys. Rev. B 94, 241406(R) (2016).

[102] C. L. Kane and M. P. A. Fisher, Transport in a One-Channel Luttinger Liquid, Phys. Rev. Lett. 68, 1220 (1992).

[103] C. L. Kane and M. P. A. Fisher, Nonequilibrium Noise and Fractional Charge in the Quantum Hall Effect, Phys. Rev. Lett. 72, 724 (1994).

[104] I. Safi and E. V. Sukhorukov, Determination of tunneling charge via current measurements, Europhys. Lett. 91, 67008 (2010).

[105] T. Giamarchi, Quantum Physics in One Dimension (Oxford University Press, Oxford, 2003).

[106] R. Guyon, P. Devillard, T. Martin, and I. Safi, Klein factors in multiple fractional quantum Hall edge tunneling, Phys. Rev. B 65, 153304 (2002).

[107] R. Hanbury Brown and R. Q. Twiss, A test of a new type of stellar interferometer on Sirius, Nature (London) 178, 1046 (1956).

[108] C. L. Kane and M. P. A. Fisher, Thermal Transport in a Luttinger Liquid, Phys. Rev. Lett. 76, 3192 (1996).

[109] L. Vannucci, F. Ronetti, G. Dolcetto, M. Carrega, and M. Sassetti, Interference-induced thermoelectric switching and heat rectification in quantum Hall junctions, Phys. Rev. B 92, 075446 (2015).

[110] F. Ronetti, L. Vannucci, G. Dolcetto, M. Carrega, and M. Sassetti, Spin-thermoelectric transport induced by interactions and spin-flip processes in two-dimensional topological insulators, Phys. Rev. B 93, 165414 (2016).

[111] D. Chevallier, J. Rech, T. Jonckheere, C. Wahl, and T. Martin, Poissonian tunneling through an extended impurity in the quantum Hall effect, Phys. Rev. B 82, 155318 (2010).

[112] G. Dolcetto, S. Barbarino, D. Ferraro, N. Magnoli, and M. Sassetti, Tunneling between helical edge states through extended contacts, Phys. Rev. B 85, 195138 (2012).

[113] G. Dolcetto, M. Sassetti, and T. L. Schmidt, Edge physics in two-dimensional topological insulators, Riv. Nuovo Cim. 39, 113 (2016).

[114] M. Moskalets, High-temperature fusion of a multielectron leviton, Phys. Rev. B 97, 155411 (2018).

[115] N. Traverso Ziani, F. Cavaliere, and M. Sassetti, Signatures of Wigner correlations in the conductance of a one-dimensional quantum dot coupled to an AFM tip, Phys. Rev. B 86, 125451 (2012).

[116] J. von Delft and H. Schoeller, Bosonization for beginners refermionization for experts, Ann. Phys. (Leipzig) 7, 225 (1998).

[117] G. B. Arfken and H. J. Weber, Mathematical Methods for Physicists, 5th ed. (Academic, San Diego, 2001).

[118] T. Needham, Visual Complex Analysis (Clarendon, Oxford, 1997). 